\title{
Thermo-convective instability in a rotating ferromagnetic fluid layer with temperature modulation
}

https://doi.org/10.1515/phys-2018-0109

Received Dec 18, 2017; accepted Sep 27, 2018

\begin{abstract}
We study the thermoconvective instability in a rotating ferromagnetic fluid confined between two parallel infinite plates with temperature modulation at the boundaries. We use weakly nonlinear stability theory to analyze the stationary convection in terms of critical Rayleigh numbers. The influence of parameters such as the Taylor number, the ratio of the magnetic force to the buoyancy force and the magnetization on the flow behaviour and structure are investigated. The heat transfer coefficient is analyzed for both the in-phase and the out-of-phase modulations. A truncated Fourier series is used to obtain a set of ordinary differential equations for the time evolution of the amplitude of convection for the ferromagnetic fluid flow. The system of differential equations is solved using a recent multi-domain spectral collocation method that has not been fully tested on such systems before. The solutions sets are presented as sets of trajectories in the phase plane. For some supercritical values of the Rayleigh number, spiralling trajectories that transition to chaotic solutions are obtained. Additional results are presented in terms of streamlines and isotherms for various Rayleigh numbers.
\end{abstract}

Keywords: Thermal instability, Ferromagnetic Fluids, Weakly nonlinear stability, Rotation, Multi-domain spectral collocation method

PACS: 47.11.Kb; 47.20.Bp

\footnotetext{
*Corresponding Author: Precious Sibanda: School of Mathematics, Statistics and Computer Science, University of KwaZulu-Natal, Private Bag X01, Scottsville, Pietermaritzburg 3209, South Africa; Email: sibandaP@ukzn.ac.za

Osman Adam Ibrahim Noreldin: School of Mathematics, Statistics and Computer Science, University of KwaZulu-Natal, Private Bag X01, Scottsville, Pietermaritzburg 3209, South Africa; Email: noreldino@ukzn.ac.za
}

\section{Introduction}

Ferromagnetic fluids are colloids consisting of nanometersized magnetic particles suspended in a fluid carrier. The magnetization of a ferromagnetic fluid depends on the temperature, the magnetic field, and the density of the fluid. The magnetic force and the thermal state of the fluid may give rise to convection currents. Studies on the flow of ferromagnetic fluids include, for example, Finalyson [1] who studied instabilities in a ferromagnetic fluid using free-free and rigid-rigid boundaries conditions. He used the linear stability theory to predict the critical Rayleigh number for the onset of instability when both a magnetic and a buoyancy force are present. The generalization of Rayleigh Benard convection under various assumption is reported by Chandrasekhar [2]. In the last few decades the study of heat transfer in ferromagnetic fluids has attracted many researchers due to the potential application of these fluids in industry, such as in the sealing of rotating shafts, ink, and so on. An authoritative introduction to research on magnetic fluids is given by Rosensweig [3].

Schwab et al. [4] studied the Finlayson problem experimentally in the case of a strong magnetic field and determined the parameters for the onset of convection. Their results were shown to be in good agreement with those of Finlayson [1]. Stiles and Kagan [5] extended the experimental problem reported by Schwab et al. [4] by introducing a strong magnetic field. A weakly nonlinear stability analysis was used by Russell et al. [6] for magnetized ferrofluids heated from above with the Rayleigh number as the control parameter for the onset of convection. They showed that heat transfer depends on the temperature difference between the bounding surfaces.

The rotation of fluids is an interesting topic that has been studied by, for example, Greenspan [7]. The classical Rayleigh-Benard problem when the fluid layer is rotating is well known in the case of ordinary viscous fluids and has been reported by Chandrasekhar [2]. However, ferromagnetic fluids are known to exhibit very peculiar characteristics when set to rotate. Demonstrating the effect of 
rotation on convection in ferromagnetic fluids is scientifically important to researchers. Gupta and Gupta [8] examined the onset of convection in a ferromagnetic fluid heated from below and rotating about a vertical axis subject to a uniform magnetic field. They concluded that overstability may not occur for Prandtl numbers smaller than unity. The thermo-convective instability in a rotating ferrofluid was further analyzed by Venkatasubramanian and Kaloni [9]. They presented both analytical and numerical results for free and rigid boundary conditions. Their results were in good agreement with those of Finlayson [1] and Chandrasekhar [2] for some limiting cases. Thermoconvection in a ferromagnetic fluid has been studied by other researchers, for instance, [10, 12].

The problem associated with convection in ferromagnetic fluids is both relevant and mathematically challenging. The unmodulated Rayleigh Benard problem of a ferromagnetic fluid has been extensively studied. The effect of a magnetic modulation on the stability of a magnetic liquid layer heated from above was studied by Aniss et al. [13]. They used the Floquet theory for their study of the onset of convection. The study showed the possibility of a competitive interaction between harmonic and subharmonic modes at the onset of convection. Convective instability in a ferromagnetic fluid layer with time-periodic modulation in the temperature field was investigated by Singh and Bajaj [14] using the linear stability theory and the classical Floquet theory. Their result agrees with those of Aniss et al. [13].

Convection in a rotating horizontal fluid layer confined in a porous medium with temperature modulation at the boundary was studied by Bhadauria [19]. He investigated the stability of the flow using the Galerkin method and the Flouquet theory. In this study we analyze thermoconvective instability in a rotating ferromagnetic fluid layer with time periodic temperature boundary conditions. The fluid layer is heated from below and rotates about the vertical axis subject to a uniform magnetic field. We assume two stress free and two rigid boundary conditions. The Ginzburg Landau equation is obtained, see [20] for details on the relevance of the Ginzburg Landau equation. Nonlinear ordinary differential equations of the Lorenz type are obtained and solved numerically using the multidomain spectral collocation method [16-18]. This method has not been fully tested before on evolution equations of this nature, hence the accuracy of solutions obtained using this method is also a matter of concern in this study. Heat transfer in the rotating horizontal fluid layer is discussed.

\section{Mathematical formulation}

Consider a ferromagnetic fluid confined between two infinite horizontal plates at $z=-h / 2$ and $z=h / 2$. The layer is heated from below and cooled from above, and is rotating uniformly about the vertical axis with constant angular velocity $\Omega$. The lower and upper plates are subjected to an oscillatory temperature $T_{0}+\Delta T\left[1+\epsilon^{2} \cos (\omega t+\varphi)\right]$ where $\omega$ is the modulation frequency and $\varphi$ is the phase angle. The Oberbeck-Boussinesq approximation is assumed to be applicable. The magnetization $\mathbf{M}$ of the ferrofluid is assumed to be parallel to the magnetic field $\mathbf{H}$. The equations describing the fluid motion under these assumptions are the continuity equation, modified momentum equation, energy equation and Maxwell's equations (Finlayson [1] and Gupta and Gupta [8]):

$$
\begin{gathered}
\nabla \cdot V=0, \\
\rho_{0} \frac{D V}{D t}=-\nabla P^{\prime}+\mu \nabla^{2} V+\rho \mathbf{g} \\
\left.+\nabla \cdot(\mathbf{H B})+2 \rho_{0} V \times \Omega\right)+\frac{\rho_{0}}{2} \nabla(|\Omega \times \mathbf{r}|), \\
{\left[\rho_{0} C_{V \cdot H}-\mu_{0} \mathbf{H} \cdot\left(\frac{\partial \mathbf{M}}{\partial T}\right)_{V, H}\right] \frac{D T}{D t}} \\
+\mu_{0} T\left(\frac{\partial \mathbf{M}}{\partial T}\right)_{V, H} \cdot \frac{D \mathbf{H}}{D t}=\kappa \nabla^{2} T+\Phi, \\
\nabla \cdot \mathbf{B}=0 ; \quad \nabla \times \mathbf{H}=0,
\end{gathered}
$$

where $V$ is the velocity field, $\rho_{0}$ is the density at the ambient temperature, $P^{\prime}=P+\frac{\mu_{0}}{2} \mathbf{H}^{2}$ is the pressure, $\mu$ is the viscosity, $\mathbf{g}$ is the gravitational body force, $\mathbf{B}$ is the magnetic induction, $\mu_{0}$ is the magnetic permeability, $T$ is the temperature, $\kappa$ is the thermal conductivity, $C_{V, H}$ is the heat capacity at constant volume and magnetic field, $\alpha$ is the thermal expansion coefficient and $\Phi$ is the viscous dissipation. The magnetization and magnetic field are related by the formula

$$
\mathbf{B}=\mu_{0}(\mathbf{H}+\mathbf{M}) .
$$

The magnetization is dependent on the temperature and magnitude of magnetic field, so that

$$
\mathbf{M}=\frac{\mathbf{H}}{H} \mathbf{M}(H, T) .
$$

Equation (6) is linearized using the Taylor expansion

$$
\mathbf{M}=\mathbf{M}_{\mathbf{0}}+\chi\left(\mathbf{H}-\mathbf{H}_{\mathbf{0}}\right)-K\left(T-T_{1}\right),
$$


where $\chi \equiv(\partial \mathbf{M} / \partial \mathbf{H})_{H_{0}, T_{1}}$ is the magnetic susceptibility and $K \equiv-(\partial \mathbf{M} / \partial T)_{H_{0}, T_{1}}$ is pryomagnetic coefficient, $H_{0}$ is the uniform magnetic field and $T_{1}=\left(T_{\infty}+T_{0}\right) / 2, T_{\infty}$ and $T_{0}$ are the temperatures at $h / 2$ and $-h / 2$, respectively. The study is restricted to the case when magnetization induced by the temperature variation is much smaller than that induced by the external magnetic field. The density varies linearly with temperature as

$$
\rho=\rho_{0}\left(1-\alpha\left(T-T_{1}\right)\right) .
$$

\section{Weakly nonlinear stability}

In this section we use weakly nonlinear stability analysis to study the evolution of disturbances in a ferromagnetic fluid with two free and two rigid boundary conditions and temperature modulation. Using Eqs. (4) and (5) and assuming that the magnetic field $\mathbf{H}$ is collinear with the magnetic induction $\mathbf{B}$, Eq. (2) reduces to

$$
\begin{aligned}
\rho_{0} \frac{D V}{D t} & =-\nabla P+\mu \nabla^{2} V+\rho \mathbf{g}+\mu_{0} \mathbf{M} \cdot \nabla \mathbf{H} \\
& \left.+2 \rho_{0} V \times \Omega\right)+\frac{\rho_{0}}{2} \nabla(|\Omega \times \mathbf{r}|) .
\end{aligned}
$$

The basic state solution of Eqs. (1)-(4) with (9) is obtained as

$$
\begin{aligned}
V & =0, \\
T_{b}(z, t) & =T_{1}+\frac{\Delta T}{2}-\beta z+\epsilon^{2}(F(z, t)),
\end{aligned}
$$

where

$$
\begin{aligned}
F(z, t) & =\operatorname{Re}\left\{\frac { \Delta T } { \operatorname { s i n h } \lambda } \left[e^{i \varphi} \sinh \lambda(1 / 2-z / h)\right.\right. \\
& \left.+\sinh \lambda(1 / 2-z / h)] e^{i \omega t}\right\}, \\
\lambda^{2} & =i \omega \rho_{0} C h^{2} / \kappa, \quad \beta=\frac{\Delta T}{h} .
\end{aligned}
$$

Following $[1,3]$ we define

$$
\mathbf{M}_{b}+\mathbf{H}_{b}=\text { constant, }
$$

and

$$
\begin{aligned}
\mathbf{H}_{b} & =\left(\mathbf{H}_{0}-\frac{\left.K\left(T_{b}-T_{1}\right)\right)}{1+\chi}\right) \hat{e}_{z}, \\
\mathbf{M}_{b} & =\left(\mathbf{M}_{0}+\frac{\left.K\left(T_{b}-T_{1}\right)\right)}{1+\chi}\right) \hat{e}_{z} .
\end{aligned}
$$

We superimpose small perturbations on the basic state. The perturbed quantities are defined as

$$
T=T_{b}+T^{\prime}, \quad \mathbf{H}_{i}=\mathbf{H}_{i}^{\prime}, \quad \mathbf{M}_{i}=\mathbf{M}_{i}^{\prime} \text { for } \mathrm{i}=1,2 ;
$$

$$
\mathbf{H}_{3}=\mathbf{H}_{b}+\mathbf{H}_{3}^{\prime}, \quad \mathbf{M}_{3}=\mathbf{M}_{b}+\mathbf{M}_{3}^{\prime},
$$

where the prime represents a perturbed quantity. The linearization of Eqs. (6) and (7) gives

$$
\begin{gathered}
\mathbf{H}_{i}^{\prime}+\mathbf{M}_{i}^{\prime}=\left(1+\frac{\mathbf{M}_{0}}{\mathbf{H}_{0}}\right) \mathbf{H}_{i}^{\prime}, \quad i=1,2 . \\
\mathbf{H}_{3}^{\prime}+\mathbf{M}_{3}^{\prime}=(1+\chi) \mathbf{H}^{\prime}-K T^{\prime} .
\end{gathered}
$$

We assume that $K \Delta T<<(1+\chi) \mathbf{H}_{0}$. For more details see [1]. Substituting Eq. (13) and using the curl operator on Eq. (9) we obtain the vorticity equation

$$
\rho_{0} \frac{\partial \zeta^{\prime}}{\partial t}=\mu \nabla^{2} \zeta^{\prime}+\rho_{0} \Omega \frac{\partial w^{\prime}}{\partial z} .
$$

Substituting Eqs. (12), (14) and (15) in Eq. (9) and using $\mathbf{H}^{\prime}=$ $\nabla \phi^{\prime}$ where $\phi$ is the magnetic potential, the $z$-component of the resulting equation can be written as,

$$
\begin{aligned}
\rho_{0} \frac{\partial}{\partial t} \nabla^{2} w^{\prime}-\mu \nabla^{4} w^{\prime} & =\rho_{0} g \alpha \nabla_{l}^{2} T^{\prime} \\
& +\frac{\mu_{0} K^{2} \beta}{1+\chi} \nabla_{l}^{2} T^{\prime}-\mu_{0} K \beta \frac{\partial}{\partial z} \nabla_{l}^{2} \phi^{\prime} \\
& -2 \rho_{0} \Omega \frac{\partial \zeta^{\prime}}{\partial z} .
\end{aligned}
$$

On using Eq. (13) in Eq. (3) and linearizing we obtain

$$
\begin{aligned}
& \rho_{0} C \frac{\partial T^{\prime}}{\partial t}+\rho_{0} C w^{\prime} \frac{\partial T^{\prime}}{\partial z}-\mu_{0} T_{0} K\left(\frac{\partial}{\partial t}\left(\frac{\partial \phi^{\prime}}{\partial z}\right)\right. \\
& \left.+w^{\prime} \frac{\partial^{2} \phi^{\prime}}{\partial z^{2}}\right)+\left(\rho_{0} C \beta-\frac{\mu_{0} T_{0} K^{2} \beta}{1+\chi}\right)\left(-1+\epsilon \frac{\partial F}{\partial z}\right) w \\
& =\nabla^{2} T^{\prime} .
\end{aligned}
$$

Finally, substituting Eqs. (14) and (15) into Eq. (4) we have

$$
(1+\chi) \frac{\partial^{2} \phi^{\prime}}{\partial z^{2}}-\left(1+\frac{\mathbf{M}_{0}}{\mathbf{H}_{0}}\right) \nabla_{l}^{2} \phi^{\prime}-K \frac{\partial T^{\prime}}{\partial z}=0,
$$

where $\rho_{o} C=\rho_{0} C_{V, H}+\mu_{0} K \mathbf{H}_{0}$ and $\nabla_{l}^{2}=\partial^{2} / \partial x^{2}+\partial^{2} / \partial y^{2}$ is the Laplace operator in two dimension. For the clarity we drop the prime from the perturbed quantities and introduce the following dimensionless variables

$$
\begin{aligned}
\left(x^{\star}, y^{\star}, z^{\star}\right) & =(x, y, x) / h, w^{\star}=w^{\prime} h / v, \\
t^{\star} & =\mu t / \rho_{0} h^{2}, \\
\zeta^{\star} & =\zeta^{\prime} h^{2} / v, \quad \theta^{\star}=\left(\kappa R a^{\frac{1}{2}} T^{\prime}\right) /\left(\rho_{0} C \beta v h\right), \\
\phi^{\star} & =\left((1+\chi) \kappa R a^{\frac{1}{2}} \phi^{\prime}\right) /\left(\rho_{0} C \beta v h^{2}\right) .
\end{aligned}
$$

The linearized perturbed Eqs. (16)-(19) in the dimensionless form can be written as,

$$
\frac{\partial}{\partial t^{\star}} \nabla^{2} w^{\star}-\nabla^{4} w^{\star}+T a^{\frac{1}{2}} \frac{\partial \zeta^{\star}}{\partial z}
$$




$$
\begin{gathered}
-R a^{\frac{1}{2}}\left(1+M_{1}\right) \nabla_{l}^{2} \theta^{\star}+R a^{\frac{1}{2}} M_{1} \frac{\partial}{\partial z} \nabla_{l}^{2} \phi^{\star}=0, \\
-T a^{\frac{1}{2}} \frac{\partial w^{\star}}{\partial z^{\star}}+\frac{\partial \zeta^{\star}}{\partial t^{\star}}-\nabla^{2} \zeta^{\star}=0, \\
\left(R a^{\frac{1}{2}}-R a^{\frac{1}{2}} M_{2}\right)\left(-1+\epsilon^{2} \frac{\partial F}{\partial z^{\star}}\right) w^{\star}+\operatorname{Pr} \frac{\partial \theta^{\star}}{\partial t^{\star}} \\
+\operatorname{Pr}^{\star} \frac{\partial \theta^{\star}}{\partial z^{\star}}-\nabla^{2} \theta^{\star}-\operatorname{Pr}_{2} \frac{\partial}{\partial t^{\star}}\left(\frac{\partial \phi^{\star}}{\partial z^{\star}}\right) \\
-\operatorname{Pr}_{2} w^{\star} \frac{\partial^{2} \phi^{\star}}{\partial z^{\star 2}}=0, \\
-\frac{\partial \theta^{\star}}{\partial z^{\star}}+\frac{\partial^{2} \phi^{\star}}{\partial z^{2}}+M_{3} \nabla_{l}^{2} \phi^{\star}=0,
\end{gathered}
$$

where $T a=\frac{4 \Omega h^{4}}{v^{2}}$ is the Taylor number, $R a=\frac{\rho_{0} C \beta \alpha g h^{4}}{v \kappa}$ is the Rayleigh number, $M_{1}=\frac{\mu_{0} K^{2} \beta}{(1+\chi) \rho_{0} \alpha g}$ is the ratio of the magnetic force to the buoyancy force, $M_{2}=\frac{\mu_{0} T_{0} K^{2}}{(1+\chi) \rho_{0} C}$ is a nondimensional parameter, $\mathrm{Pr}=\frac{\mu C}{\kappa}$ is the Prandtl number and $M_{3}=\left(1+\frac{\mathbf{M}_{0}}{\mathbf{H}_{0}}\right) /(1+\chi)$ is a measure of nonlinearity of the magnetization. The magnetic Rayleigh number can be obtained from the formula $N=R a M_{1}$. Hereafter the asterisk will be dropped from Eqs. (21)-(24).

The associated boundary conditions for the system of Eqs. (21)-(24) are

- Free boundary conditions

$$
\begin{aligned}
& w=0, D^{2} w=0, D \zeta=0, \theta=0, D \phi=0, \\
& \text { at } z= \pm \frac{1}{2} .
\end{aligned}
$$

- Rigid boundary conditions

$$
\begin{aligned}
& w=0, D w=0, \zeta=0, \theta=0, \\
& \text { at } z= \pm \frac{1}{2},
\end{aligned}
$$

where

$$
D=\partial / \partial z
$$

\subsection{The solution for stress free boundaries}

The solution for stress free boundaries has been discussed in $[1,3,9]$. Here we only emphasize the solution aspects which have not been discussed before. We solve the eigenvalue problem with two stress free boundaries to study the onset of instability in the ferromagnetic fluid. We consider a small variation in time scale $\tau=\epsilon^{2} t$ such that stationary convection occurs at lower orders of $\epsilon$ and introduce the following asymptotic expansions

$$
R a^{\frac{1}{2}}=\left(R a^{\alpha}\right)^{\frac{1}{2}}+\epsilon^{2} R a_{2}^{\frac{1}{2}}+\epsilon^{4} R a_{4}^{\frac{1}{2}}+\cdots,
$$

$$
\begin{gathered}
w=\epsilon w_{1}+\epsilon^{2} w_{2}+\epsilon^{3} w_{3}+\cdots, \\
\zeta=\epsilon \zeta_{1}+\epsilon^{2} \zeta_{2}+\epsilon^{3} \zeta_{3}+\cdots, \\
\theta=\epsilon \theta_{1}+\epsilon^{2} \theta_{2}+\epsilon^{3} \theta_{3}+\cdots, \\
\phi=\epsilon \phi_{1}+\epsilon^{2} \phi_{2}+\epsilon^{3} \phi_{3}+\cdots .
\end{gathered}
$$

Substituting Eqs. (27)-(31) into Eqs. (21)-(24), at the lowest order of $\epsilon$ we obtain

$$
B Z_{1}=R_{1},
$$

where

$$
B=\left(\begin{array}{cccc}
-\nabla^{4} & \sqrt{T a} \frac{\partial}{\partial z} & -\sqrt{R a^{\alpha}}\left(1+M_{1}\right) \nabla_{l}^{2} & \sqrt{R a^{\alpha}} M_{1} \frac{\partial}{\partial z} \nabla_{l}^{2} \\
-\sqrt{T a} \frac{\partial}{\partial z} & -\nabla^{2} & 0 & 0 \\
-\sqrt{R a^{\alpha}}\left(1-M_{2}\right) & 0 & -\nabla^{2} & 0 \\
0 & 0 & -\frac{\partial}{\partial z} & \frac{\partial^{2}}{\partial z^{2}}+M_{3} \nabla_{l}^{2}
\end{array}\right),
$$

$Z_{1}=\left(\begin{array}{c}w_{1} \\ \zeta_{1} \\ \theta_{1} \\ \phi_{1}\end{array}\right)$ and $R_{1}=\left(\begin{array}{l}0 \\ 0 \\ 0 \\ 0\end{array}\right)$. This equation corresponds to the linear equations in $[1,9]$. Solving Eq. (32) we obtain the solution

$$
w_{1}=A(\tau) \sin a x \cos \pi z,
$$

$$
\zeta_{1}=-\frac{T a^{\frac{1}{2}} \pi}{\pi^{2}+a^{2}} A(\tau) \sin a x \sin \pi z
$$

$$
\theta_{1}=\frac{\left(R a^{\alpha}\right)^{\frac{1}{2}}\left(1-M_{2}\right)}{\pi^{2}+a^{2}} A(\tau) \sin a x \cos \pi z,
$$

$$
\phi_{1}=-\left(\frac{\left(R a^{\alpha}\right)^{\frac{1}{2}}\left(1-M_{2}\right) \pi}{\left(\pi^{2}+a^{2}\right)\left(\pi^{2}+a^{2} M_{3}\right)}\right)
$$

$$
A(\tau) \sin a x \sin \pi z,
$$

where $a$ is a dimensionless wave number. Thus, the stationary Rayleigh number is given as

$$
R a^{\alpha}=\frac{\left(\left(\pi^{2}+a^{2}\right)^{3}+\pi^{2} T a\right)\left(\pi^{2}+a^{2} M_{3}\right)}{(1-M 2)\left(a^{2} \pi^{2} M_{1}+a^{2}\left(M_{1}+1\right)\left(\pi^{2}+a^{2} M_{3}\right)\right)} .
$$

To find the critical wave number and the corresponding critical Rayleigh number we set $a^{2}=\pi^{2} x$. Then the stationary Rayleigh number can be written as

$$
R a^{\alpha}=\frac{\left(\pi^{4}(1+x)^{3}+T a\right)\left(1+x M_{3}\right)}{(1-M 2) x\left(1+x M_{3}\left(M_{1}+1\right)\right)} .
$$


This result agrees with $[1,3,9]$. Since $M_{2}$ is very small as indicated in $[1,9]$ it can be neglected in the subsequent analysis.

The critical wave number and the corresponding critical Rayleigh number are obtained from

$$
\frac{\partial R a^{\alpha}}{\partial x}=0
$$

then we have

$$
\begin{aligned}
& -T a-2 T a\left(x M_{3}+x M_{1} M_{3}\right) \\
& -T a\left(x^{2} M_{3}^{2}+x^{2} M_{1} M_{3}^{2}\right) \\
& +\pi^{4}(1+x)^{2}\left(-1+2 x-2 x M_{3}-2 x M_{1} M_{3}-4 x^{2} M_{3}\right. \\
& \left.+x^{2} M_{3}^{2} M_{1}+2 x^{3} M_{3}^{2}+2 x^{3} M_{1} M_{3}^{2}\right)=0 .
\end{aligned}
$$

For the case $T a=0, M_{1}=0$ and $M_{3}=0$, the classical critical wave number is

$$
a_{c}=\frac{\pi}{\sqrt{2}}
$$

with corresponding classical critical Rayleigh number

$$
R a_{c}^{\alpha}=\frac{27}{4} \pi^{4} .
$$

The magnetic Rayleigh number is also of an interest and can be expressed as

$$
N^{\alpha}=R a^{\alpha} M_{1}=\frac{\left(\pi^{4}(1+x)^{3}+T a\right) M_{1}\left(1+x M_{3}\right)}{x\left(1+x M_{3}\left(M_{1}+1\right)\right)} .
$$

For large values of $M_{1}$ the magnetic Rayleigh number in the absence of buoyancy effects is obtained as

$$
N^{\alpha}=\frac{\left(\pi^{4}(1+x)^{3}+T a\right)\left(1+x M_{3}\right)}{x^{2} M_{3}} .
$$

The critical wave number and corresponding critical magnetic Rayleigh number are obtained from solving the equation

$$
-2 T a+M 3 x+\pi^{4}(1+x)^{2}\left(-2+x-M 3 x+2 M 3 x^{2}\right)=0 .
$$

The critical wave number and corresponding Rayleigh numbers are given for different values of the Taylor number Ta in Tables 1 and 2 .

At second order $O\left(\epsilon^{2}\right)$ we obtain the following equations:

$$
B Z_{2}=R_{2}
$$

$$
\text { where } Z_{2}=\left(\begin{array}{c}
w_{2} \\
\zeta_{2} \\
\theta_{2} \\
\phi_{2}
\end{array}\right) \text { and } R_{2}=\left(\begin{array}{l}
R_{21} \\
R_{22} \\
R_{23} \\
R_{24}
\end{array}\right) \text { with }
$$$$
R_{21}=0, \quad R_{22}=0, R_{24}=0 \text {, }
$$

Table 1: Comparison of the critical wave number and corresponding Rayleigh number for $M_{1}=1$ and $M_{3}=5$.

\begin{tabular}{ccccc}
\hline & \multicolumn{2}{c}{ Present study } & \multicolumn{2}{c}{ Ref [9] } \\
\hline$T a$ & $a_{c}$ & $R a_{c}^{\alpha}$ & $a_{c}$ & $R a_{c}^{\alpha}$ \\
1 & 2.11212 & 269.6382 & 2.7348 & 461.5368 \\
10 & 2.15849 & 275.0998 & 3.7827 & 883.4022 \\
100 & 2.49953 & 322.9328 & 5.7215 & 2727.2022 \\
1000 & 3.65242 & 608.4028 & 8.6174 & 10619.856 \\
10000 & 5.66466 & 1862.582 & 12.8255 & 45606.430 \\
\hline
\end{tabular}

Table 2: Comparison of the critical wave number and corresponding Magnetic Rayleigh number for $M_{1} \rightarrow \infty$ and $M_{3}=3$.

\begin{tabular}{ccccc}
\hline & \multicolumn{2}{c}{ Present study } & \multicolumn{2}{c}{ Ref [9] } \\
\hline$T a$ & $a_{c}$ & $N_{c}^{\alpha}$ & $a_{c}$ & $N_{c}^{\alpha}$ \\
1 & 2.6084 & 880.7959 & 2.7348 & 1037.8896 \\
10 & 2.62069 & 897.4541 & 3.8770 & 1885.6592 \\
100 & 2.72574 & 1050.072 & 5.7736 & 5617.3574 \\
1000 & 3.2225 & 2083.258 & 8.6487 & 21520.978 \\
10000 & 4.28305 & 7315.093 & 12.8455 & 91759.273 \\
\hline
\end{tabular}

$$
R_{23}=-\frac{\operatorname{Pr} \pi\left(R a^{\alpha}\right)^{\frac{1}{2}}}{2\left(\pi^{2}+a^{2}\right)} A^{2}(\tau) \sin ^{2} a x \sin 2 \pi z .
$$

The solution at the second order is

$$
w_{2}=0, \quad \zeta_{2}=0,
$$

$$
\begin{aligned}
& \theta_{2}=-\left(\frac{\operatorname{Pr} \pi\left(R a^{\alpha}\right)^{\frac{1}{2}}}{4\left(\pi^{2}+a^{2}\right)\left(2 \pi^{2}+a^{2}\right)}\right) \\
& A^{2}(\tau) \sin ^{2} a x \sin 2 \pi z,
\end{aligned}
$$

$$
\begin{aligned}
& \phi_{2}=-\left(\frac{P r \pi^{2}\left(R a^{\alpha}\right)^{\frac{1}{2}}}{4\left(2 \pi^{2}+a^{2}\right)\left(\pi^{2}+a^{2}\right)\left(2 \pi^{2}+a^{2} M_{3}\right)}\right) \\
& A^{2}(\tau) \sin ^{2} a x \cos 2 \pi z .
\end{aligned}
$$

At the third order, we obtain

$$
B Z_{3}=R_{3} \text {, }
$$

where $Z_{3}=\left(\begin{array}{c}w_{3} \\ \zeta_{3} \\ \theta_{3} \\ \phi_{3}\end{array}\right)$ and $R_{2}=\left(\begin{array}{l}R_{31} \\ R_{32} \\ R_{33} \\ R_{34}\end{array}\right)$ with

$$
\begin{array}{r}
R_{31}=\left\{-\gamma^{2} \frac{d A}{d \tau}+\frac{a^{2}\left(R a^{\alpha}\right)^{\frac{1}{2}} R_{a 2}^{\frac{1}{2}}\left(1+M_{1}\right)}{\gamma^{2}\left(\pi^{2}+a^{2} M_{3}\right)}\right. \\
\left.\left(\left(1+M_{1}\right) \pi^{2}+a^{2} M_{3}\right) A(\tau)\right\} \sin a x \cos \pi z,
\end{array}
$$




$$
\begin{gathered}
R_{32}=-\frac{T a^{\frac{1}{2}} \pi^{2}}{\gamma^{2}} \frac{d A}{d \tau} \sin a x \sin \pi z, \\
R_{33}=-\left(\left(\left(R a^{\alpha}\right)^{\frac{1}{2}} \frac{\partial F}{\partial z}-R a_{2}^{\frac{1}{2}}\right) A(\tau)\right. \\
\left.+\frac{P r\left(R a^{\alpha}\right)^{\frac{1}{2}}}{\gamma^{2}} \frac{d A}{d \tau}\right) \sin a x \cos \pi z+\frac{P^{2} \pi^{2}\left(R a^{\alpha}\right)^{\frac{1}{2}}}{2 \gamma^{2}\left(2 \pi^{2}+a^{2}\right)} \\
A^{3}(\tau) \sin ^{3} a x \cos \pi z \cos 2 \pi z,
\end{gathered}
$$

$$
R_{34}=0 .
$$

Here, $\gamma^{2}=a^{2}+\pi^{2}$. To obtain the Ginzburg Landau equation we applied the Fredholm solvability condition $[11,18]$

$$
\int_{0}^{\frac{1}{2}} \int_{0}^{\frac{2 \pi}{a}}\left[\hat{w}_{1} R_{31}+\hat{\zeta}_{1} R_{32}+\hat{\theta} R_{33}\right] d x d z=0,
$$

where $\hat{w}_{1}, \hat{\zeta}_{1}$ and $\hat{\theta}_{1}$ are the solutions of the adjoint system of the first order. This gives

$$
\begin{aligned}
& {\left[\frac{\pi \gamma^{2}}{4 a}+\frac{P r R a^{\alpha} \pi}{4 a \gamma^{4}}-\frac{T a \pi^{4}}{4 a \gamma^{4}}\right] \frac{d A}{d \tau}=\left\{\frac{\pi R a^{\alpha \frac{1}{2}} R a_{2}^{\frac{1}{2}}}{4 a \gamma^{2}}\right.} \\
& -\frac{\pi R a^{\alpha}}{a \gamma^{2}} \mathbf{I}+\frac{a \pi R a^{\alpha \frac{1}{2}} R a_{2}^{\frac{1}{2}}\left(1+M_{1}\right)}{4 \gamma^{2}\left(\pi^{2}+a^{2} M_{3}\right)} \\
& \left.\left(\left(1+M_{1}\right) \pi^{2}+a^{2} M_{3}\right)\right\} A-\frac{3 P r^{2} \pi^{3} R a^{\alpha}}{64 a \gamma^{4}\left(2 \pi^{2}+a^{2}\right)} A^{3} .
\end{aligned}
$$

The above equation reduces to

$$
\frac{d A}{d \tau}=\Delta_{1} A-\Delta_{2} A^{3},
$$

where $\Delta_{1}=\gamma^{2} /\left(\gamma^{6}+P r R a^{\alpha}-T a \pi^{3}\right)$ $\left(R a^{\star}-4 R a^{\alpha} \mathbf{I}+\frac{a^{2} R a^{*}\left(1+M_{1}\right)\left(\left(1+M_{1}\right) \pi^{2}+a^{2} M_{3}\right)}{\pi^{2}+a^{2} M_{3}}\right)$, $R a^{*}=R a^{\alpha \frac{1}{2}} R a_{2}^{\frac{1}{2}}, \Delta_{2}=3 \operatorname{Pr}^{2} \pi^{3} R a^{\alpha} /\left(16\left(2 \pi^{2}+a^{2}\right)\right)$, and

$$
\mathbf{I}=\int_{0}^{\frac{1}{2}} \frac{d F}{d z} \cos ^{2}(\pi z) d z
$$

In this study we are also interested in heat transfer in ferromagnetic fluids. The Nusselt number for ferromagnetic fluids is defined as

$$
\begin{aligned}
N u(\tau) & =\frac{\text { Heat transfer by conduction }+ \text { convection }}{\text { Heat transfer by conduction }} \\
& =1+\frac{P r \pi^{2}\left(R a^{\alpha}\right)^{\frac{1}{2}}}{4 \gamma^{2}\left(2 \pi^{2}+a^{2}\right)} A^{2}(\tau) .
\end{aligned}
$$

\subsection{The general Lorentz type equations}

We restrict the analysis to the case of two-dimensional disturbances so that all physical quantities are independent of $y$. Using the stream function defined by

$$
u=\frac{\partial \psi}{\partial z} \text { and } w=-\frac{\partial \psi}{\partial x}
$$

equations (1)-(4) reduce to

$$
\begin{gathered}
\frac{\partial}{\partial t} \nabla_{L}^{2} \psi-\left|\mathcal{J}\left(\psi, \nabla_{L}^{2} \psi\right)\right|=\nabla_{L}^{4} \psi-M_{1} R a^{\alpha \frac{1}{2}} \frac{\partial^{2} \phi}{\partial x \partial z} \\
-R a^{\alpha \frac{1}{2}}\left(1+M_{1}\right) \frac{\partial \theta}{\partial x}+T a^{\frac{1}{2}} \frac{\partial \zeta}{\partial z} \\
\frac{\partial \zeta}{\partial t}+|\partial(\psi, \zeta)|=\nabla_{L}^{2} \zeta-T a^{\frac{1}{2}} \frac{\partial \psi}{\partial z}, \\
\operatorname{Pr}\left(\frac{\partial \theta}{\partial t}+|\mathcal{\partial}(\psi, \theta)|\right)=\nabla_{L}^{2} \theta-R a^{\alpha \frac{1}{2}} \frac{\partial \psi}{\partial x}, \\
\frac{\partial^{2} \phi}{\partial z^{2}}+M_{3} \frac{\partial^{2} \phi}{\partial x^{2}}=\frac{\partial \theta}{\partial z},
\end{gathered}
$$

where $\mathcal{\partial}$ is the Jacobian matrix. The solution of Eqs. (57)(60) represented as a minimal double Fourier series of modes $(1,1)$ for the stream function and magnetic potential and modes $(0,2)$ and $(1,1)$ for temperature and vorticity of the finite amplitude convection of the ferromagnetic fluid flows as

$$
\psi={ }_{11} \sin a x \sin \pi z
$$

$$
\begin{gathered}
\theta=B_{11} \cos a x \sin \pi z+B_{02} \sin 2 \pi z, \\
\zeta=C_{11} \sin a x \cos \pi z+C_{02} \sin 2 \pi z, \\
\phi=D_{11} \cos a x \cos \pi z,
\end{gathered}
$$

where $A_{11}, B_{11}, B_{02}, C_{11}, C_{02}$ and $D_{11}$ are time $t$ dependent amplitudes. This is equivalent to a truncated Galerkin method. Substituting and integrating over the domain, we obtain a set of four ordinary differential equations for the time evolution of the amplitudes of convection of a ferromagnetic fluid in the form

$$
\begin{gathered}
\frac{d A_{11}}{d t}=-\gamma^{2} A_{11}-\frac{a \pi R a^{\alpha \frac{1}{2}}}{\gamma^{2}\left(\pi^{2}+a_{3}^{M}\right)} B_{11} \\
-\frac{a R a^{\alpha \frac{1}{2}}\left(1+M_{1}\right)}{\gamma^{2}} B_{11}+\frac{\pi T a^{\frac{1}{2}}}{\gamma^{2}} C_{11}, \\
\frac{d C_{11}}{d t}=-\gamma^{2}-\pi T a^{\frac{1}{2}} A_{11},
\end{gathered}
$$

$$
\frac{d B_{11}}{d t}=\frac{a R a^{\alpha \frac{1}{2}}}{\operatorname{Pr}} A_{11}-\frac{\gamma^{2}}{\operatorname{Pr}} B_{11}-a \pi A_{11} B_{02},
$$




$$
\frac{d B_{02}}{d t}=\frac{a \pi}{2} A_{11} B_{11}-\frac{4 \pi^{2}}{\operatorname{Pr}} B_{02} .
$$

To simplify the equations we introduce new variables

$$
\begin{aligned}
& X_{1}=\frac{a \pi}{\gamma^{2}} A_{11}, X_{2}=\frac{-a^{2} \pi R a^{\alpha \frac{1}{2}}}{\gamma^{6}} B_{11}, \tau=\gamma^{2} t, \\
& X_{3}=\frac{-a^{2} \pi R a^{\alpha \frac{1}{2}}}{\gamma^{6}} B_{02} \text { and } X_{4}=\frac{a \pi^{2} T a^{\frac{1}{2}}}{\gamma^{6}} C_{11} .
\end{aligned}
$$

This reduces Eqs. (65)-(68) to general Lorentz type equations

$$
\begin{gathered}
\frac{d X_{1}}{d \tau}=-X_{1}+K X_{2}+X_{4}, \\
\frac{d X_{2}}{d \tau}=R X_{1}-\operatorname{Pr}^{-1} X_{2}-X_{1} X_{3}, \\
\frac{d X_{3}}{d \tau}=\frac{1}{2} X_{1} X_{2}-b \operatorname{Pr}^{-1} X_{3}, \\
\frac{d X_{4}}{d \tau}=-\operatorname{TaX}_{1}-X_{4},
\end{gathered}
$$

where $R=\frac{a^{2}}{\gamma^{6}} R a^{\alpha}, b=\frac{4 \pi^{2}}{\gamma^{2}}$, and $K=\frac{\left(2 \pi^{2}+a^{2} M_{3}\right)+M_{1}\left(\pi^{2}+a^{2} M_{3}\right)}{\pi^{2}+a^{2} M_{3}}$.

\subsection{Stability of Lorentz equations}

In this section we discuss the stability of the nonlinear systems of differential equations that describe the evolution of the convection amplitudes for a ferromagnetic fluid flow. Firstly, we note that the nonlinear Eqs. (71)-(74) are invariant under the transformation

$$
S\left(X_{1}, X_{2}, X_{3}, X_{4}\right)=\left(-X_{1},-X_{2},-X_{3},-X_{4}\right) .
$$

These equations are also uniformly bounded and dissipative in the phase space

$$
\sum_{i=1}^{4} \frac{\partial \dot{X}_{i}}{X_{i}}=-\left[1+P r^{-1}+b P r^{-1}\right]<0
$$

Thus the volume of the phase space moving with the flow for time $\tau>0$ is given by

$$
V(t)=V(0) \exp \left(-\left[1+\operatorname{Pr}^{-1}+b P r^{-1}\right] \tau\right) .
$$

We find that the stationary points of the system of nonlinear Eqs. (71)-(74) are:

- The motionless conduction solutions $(0,0,0,0)$.
- The steady solution represented by the point

$$
\begin{aligned}
& \left(x_{1}^{\star}, x_{2}^{\star}, x_{3}^{\star}, x_{4}^{\star}\right)=\left( \pm \sqrt{\frac{2 b(\operatorname{Pr} K R-(T a+1))}{\operatorname{Pr}^{2}(1+T a)},}\right. \\
& \pm \frac{T a+1}{K} \sqrt{\frac{2 b(\operatorname{Pr} K R-(T a+1))}{\operatorname{Pr}^{2}(T a+1)}}, \frac{k P r R-(T a+1)}{k P r}, \\
& \left.\mp \sqrt{\frac{2 b\left(\operatorname{Pr} K R^{2}-(T a+1)\right)}{T^{2} \operatorname{Pr}^{2}(1+T a)}}\right) .
\end{aligned}
$$

The stability of the stationary point associated with the motionless solution $X^{\star}=(0,0,0,0)$ is determined by roots of the following characteristic polynomial equation

$$
P(\xi)=\xi^{3}+d_{1} \xi^{2}+d_{2} \xi+d_{3}=0,
$$

where

$$
d_{1}=\operatorname{Pr}^{-1}, \quad d_{2}=T a-1-K R
$$

and

$$
d_{3}=\frac{T a-\operatorname{Pr} K R-1}{\operatorname{Pr}}
$$

for the eigenvalues $\xi_{i},(i=2,3,4)$ and $\xi_{1}=-\frac{b}{P r}$. It is clear that $\xi_{1}$ is always negative as $\mathrm{Pr}>0$. The remaining eigenvalues are obtained from Eq. (78), and using the RouthHurwitz criteria [21], the polynomial Eq. (78) has negative real roots if and only if

$$
R<\frac{T a-1}{K P r}
$$

and $\operatorname{Pr}>1$. This implies that the stationary solution is a stable node. Hence the critical value of $R$ where the stationary solution of ferromagnetic fluid flow loses stability and steady convective flow takes over is

$$
R=\frac{T a-1}{K P r} .
$$

The stability of the stationary point corresponding to the steady convective flow is determined by the roots of the characteristic equation

$$
p(\xi)=\xi^{4}+c_{1} \xi^{3}+c_{2} \xi^{2}+c_{3} \xi+c_{4}=0,
$$

where

$$
\begin{aligned}
c_{1} & =2 P r+2 b P r, \\
c_{2} & =2 b-2 \operatorname{Pr}^{2}-2 \operatorname{SPr}^{2} R+2 \operatorname{Pr}^{2} T a \\
& +\operatorname{Pr}^{2} x_{1}^{\star 2}+2 K \operatorname{Pr}^{2} x_{3}^{\star}, \\
c_{3} & =-2 \operatorname{Pr}-2 b P r-2 b K \operatorname{Pr} R-2 K \operatorname{Pr}^{2} R+2 \operatorname{PrTa} \\
& +2 b P r T a+K \operatorname{Pr}^{3} x_{1}^{\star} x_{2}^{\star}+2 b K \operatorname{Pr}_{3}^{\star}+2 K \operatorname{Pr}^{2} x_{3}^{\star}, \\
c_{4} & =-2 b-2 b K \operatorname{Pr} R+2 b T a-\operatorname{Pr}^{2} x_{1}^{\star 2}+\operatorname{Pr}^{2} \operatorname{Tax}_{1}^{\star 2} \\
& +\operatorname{KPr}^{2} x_{1}^{\star} x_{2}^{\star}+2 b K \operatorname{Pr}_{3}^{\star} .
\end{aligned}
$$


Applying the Routh-Hurwitz criteria to Eq. (79), it is clear that $c_{1}>0$, and $c_{3}>0$ if and only if

$$
T a+1<\operatorname{PrKR}
$$

and

$$
R<-\left(\frac{b+1}{b K+P r}\right) .
$$

Also, $c_{4}>0$ if and only if

$$
x_{1}^{\star 2}<-\left(\frac{2 b(1+\operatorname{Pr} K R)}{\operatorname{Pr}^{2}}\right)
$$

with

$$
T a+1<\operatorname{PrKR} .
$$

Hence the fixed point is stable if the condition

$$
\begin{aligned}
& \operatorname{Pr}^{3} c_{3}^{\star}+\operatorname{Pr}^{2} c_{2}^{\star}+\operatorname{Pr}_{1}^{\star}+c_{0}^{\star} \\
& >\operatorname{Pr}^{3} d_{3}^{\star}+\operatorname{Pr}^{2} d_{2}^{\star}+\operatorname{Pr}_{1}^{\star}+d_{0}^{\star},
\end{aligned}
$$

is satisfied where

$$
\begin{aligned}
c_{3}^{\star} & =\left(8 K R+8 K^{2} R^{2}+4 K \operatorname{Tax}_{1}^{\star} x_{2}^{\star}\right. \\
& +2 K x_{1}^{\star 3} x_{2}^{\star}+4 K x_{1}^{\star 2} x_{3}^{\star}+4 K^{2} x_{1}^{\star} x_{2}^{\star} x_{3}^{\star} \\
& \left.+8 K^{2} x_{3}^{\star 2}+8 K \operatorname{Tax}_{3}^{\star}\right)(1+b), \\
c_{2}^{\star} & =8(K R+1)+16 b+8 b^{2}+8 K R(3+2 b) \\
& +8 K^{2} R^{2}(b+1)+8 T a^{2}\left(b^{2}+2 b+1\right) \\
& +8 K T a x_{3}^{\star}\left(2 b^{2}+3 b+1\right)+\left(4 K x_{1}^{\star 2} x_{3}^{\star}\right. \\
& \left.+8 K^{2} x_{3}^{\star 2}\right)\left(b+b^{2}\right)+4 K^{2} R\left(x_{1}^{\star 2} x_{2}^{\star}+2 x_{3}^{\star}\right), \\
c_{1}^{\star} & =8 K R\left(b^{3}+b^{2}\right)+4 K x_{1}^{\star 2} x_{2}^{\star}\left(2 b+b^{2}\right) \\
& +\left(8 K x_{3}^{\star}+8 K R T a+4 K^{2} R x_{1}^{\star 2} x_{2}^{\star}\right)(1+b) \\
& +16 b K^{2} R x_{3}^{\star}, \quad c_{0}^{\star}=8 b+4 b^{2}+8 T a\left(b^{2}\right. \\
& +2 b+1)+8 K x_{3}^{\star}\left(b^{3}+2 b^{2}+b\right) \\
& +8 K R T a\left(b^{2}+b\right)+8 b^{2} K^{2} R x_{3}^{\star},
\end{aligned}
$$

and

$$
\begin{aligned}
d_{3}^{\star} & =\left(8 K R T a+4 K R x_{1}^{\star 2}+4\left(K+K^{2}\right) x_{1}^{\star 2} x_{2}^{\star}\right. \\
& \left.+8 K x_{3}^{\star}+16 K^{2} R x_{3}^{\star}\right)(1+b), \\
d_{2}^{\star} & =\left(16 T a+4 K x_{1}^{\star} x_{2}^{\star}\right)\left(b^{2}+2 b+1\right) \\
& +\left(8 K R T a+8 K x_{3}^{\star}\right)\left(2 b^{2}+3 b+1\right) \\
& +16 K^{2} R x_{3}^{\star}\left(b^{2}+b\right)+K^{2}\left(x_{1}^{\star 2} x_{2}^{\star 2}+4 x_{1}^{\star} x_{2}^{\star} x_{3}^{\star}+4 x_{3}^{\star}\right), \\
d_{1}^{\star} & =8 K x_{3}^{\star}\left(b^{3}+b^{2}\right)+\left({ }^{\star} T a x_{3}^{\star}+4 K \operatorname{Tax}_{1}^{\star} x_{2}^{\star}\right. \\
& \left.+8 K^{2} R^{2}\right)(b+1)+4 b K^{2}\left(x_{1}^{\star} x_{2}^{\star} x_{3}^{\star}+2 x_{3}^{\star 2}\right), \\
d_{0}^{\star} & \left.=8 K R\left(b^{3}+2 b^{2}+b\right)+4 \operatorname{Ta}^{2} b^{2}+2 b+1\right) \\
& +8 K T a x_{3}^{\star}\left(b^{2}+b\right)+4 b^{2} K^{2} x_{3}^{\star 2} .
\end{aligned}
$$

\subsection{The method of solution}

In this section, we describe the multi-domain spectral collocation method [15-18] used to obtain the solutions to Eqs. (71)-(74). The multi-domain technique assumes that the interval $\Lambda=[0, T]$ can be decomposed into $p$ non overlapping sub-intervals. The sub-intervals are defined as

$$
\Lambda_{i}=\left[\tau_{i-1}, \tau_{i}\right], \quad i=1,2, \cdots, p .
$$

In each sub-interval, the system of Eqs. (71)-(74) is written in the form

$$
\begin{aligned}
& \dot{X}_{1, s+1}^{i}+\alpha_{1,1} X_{1, s+1}^{i}+\alpha_{1,2} X_{2, s}^{i}+\alpha_{1,3} X_{3, s}^{i} \\
& +\alpha_{1,4} X_{4, s}^{i}+f_{1}\left(X_{2, s}^{i}, X_{3, s}^{i}, X_{4, s}^{i}\right)=g_{1} \\
& \dot{X}_{2, s+1}^{i}+\alpha_{2,1} X_{1, s+1}^{i}+\alpha_{2,2} X_{2, s+1}^{i}+\alpha_{2,3} X_{3, s}^{i} \\
& +\alpha_{2,4} X_{4, s}^{i}+f_{2}\left(X_{1, s+1}^{i}, X_{3, s}^{i}, X_{4, s}^{i}\right)=g_{2} \\
& \dot{X}_{3, s+1}^{i}+\alpha_{3,1} X_{1, s+1}^{i}+\alpha_{3,2} X_{2, s+1}^{i}+\alpha_{3,3} X_{3, s+1}^{i} \\
& +\alpha_{3,4} X_{4, s}^{i}+f_{3}\left(X_{1, s+1}^{i}, X_{2, s+1}^{i}, X_{4, s}^{i}\right)=g_{3} \\
& \dot{X}_{4, s+1}^{i}+\alpha_{4,1} X_{1, s+1}^{i}+\alpha_{4,2} X_{2, s+1}^{i}+\alpha_{4,3} X_{3, s+}^{i} \\
& +\alpha_{4,4} X_{4, s+1}^{i}+f_{4}\left(X_{1, s+1}^{i}, X_{2, s+1}^{i}, X_{3, s+1}^{i}\right)=g_{4},
\end{aligned}
$$

with initial conditions

$$
X_{n, S+1}^{i}\left(\tau_{i-1}\right)=X_{n}^{i-1}\left(\tau_{i-1}\right), \quad n=1,2,3,4 .
$$

Here $\alpha_{n, k}$ and $g_{n}(n, k=1,2,3,4)$ are constants while $f_{n}$ is the nonlinear component of each equation. Each subinterval $\Lambda_{i}$ is transformed to $[-1,1]$ using the transformation

$$
\tau=\frac{\tau_{i-1}-\tau_{i}}{2} t^{\star}+\frac{\tau_{i-1}+\tau_{i}}{2}, t^{\star} \in[-1,1] .
$$

The Chebyshev-Gauss-Lobatto collocation points are used to discretize the unknown functions

$$
t_{j}^{\star i}=\cos \left(\frac{\pi j}{N}\right), j=0,1, \cdots, N .
$$

The derivative of the unknown function at the collocation point is given by

$$
\frac{d X_{n, s+1}^{i}}{d \tau}\left(\tau^{i}\right)=\sum_{k=0}^{N} \mathbf{D}_{j k} X_{n, s+1}^{i}\left(t_{k}^{\star i}\right)=\mathbf{D} \mathbf{X}_{n, s+1}^{i}
$$

where

$$
\mathbf{D}=2 D / \delta \tau_{i}
$$

with $\delta \tau_{i}=\tau_{i-1}-\tau_{i}$ and $D$ is Chebyshev differentiation matrix. The vector functions at the collocation points are

$$
\mathbf{X}_{n, s+1}^{i}=\left(X_{n, s+1}^{i}\left(t_{0}^{\star i}\right), \cdots, X_{n, s+1}^{i}\left(t_{N}^{\star i}\right)\right)^{T} .
$$


Substituting Eq. (86) into Eq. (82) and reducing to matrix form we obtain the system

$$
\mathbf{A}_{n} \mathbf{X}_{n, s+1}^{i}=\mathbf{R}_{n}^{i}
$$

with $\mathbf{A}_{n}=\mathbf{D}+\alpha_{n, n} \mathbf{I}$ and

$$
\begin{aligned}
\mathbf{R}_{1}^{i}=\mathbf{g}_{1}-\left[\alpha_{1,2} \mathbf{X}_{2, s}^{i}+\alpha_{1,3} \mathbf{X}_{3, s}^{i}+\alpha_{1,4} \mathbf{X}_{4, s}^{i}\right. \\
\left.+f_{1}\left(\mathbf{X}_{2, s}^{i}, \mathbf{X}_{3, s}^{i}, \mathbf{X}_{4, s}^{i}\right)\right] \\
\mathbf{R}_{2}^{i}=\mathbf{g}_{2}-\left[\alpha_{2,1} \mathbf{X}_{1, s+1}^{i}+\alpha_{2,3} \mathbf{X}_{3, s}^{i}+\alpha_{2,4} \mathbf{X}_{4, s}^{i}\right. \\
\left.+f_{2}\left(\mathbf{X}_{1, s+1}^{i}, \mathbf{X}_{3, s}^{i}, \mathbf{X}_{4, s}^{i}\right)\right] \\
\mathbf{R}_{3}^{i}=\mathbf{g}_{3}-\left[\alpha_{3,1} \mathbf{X}_{1, s+}^{i}+\alpha_{3,2} \mathbf{X}_{2, s+1}^{i}+\alpha_{3,4} \mathbf{X}_{4, s}^{i}\right. \\
\left.+f_{3}\left(\mathbf{X}_{1, s+1}^{i}, \mathbf{X}_{2, s+1}^{i}, \mathbf{X}_{4, s}^{i}\right)\right] \\
\mathbf{R}_{4}^{i}=\mathbf{g}_{4}-\left[\alpha_{4,1} \mathbf{X}_{1, s+1}^{i}+\alpha_{4,2} \mathbf{X}_{2, s+1}^{i}+\alpha_{4,3} \mathbf{X}_{3, s+1}^{i}\right. \\
\left.+f_{4}\left(\mathbf{X}_{1, s+1}^{i}, \mathbf{X}_{2, s+1}^{i}, \mathbf{X}_{3, s+1}^{i}\right)\right]
\end{aligned}
$$

where $\mathbf{g}_{n}$ is $g_{n}$ multiplied by a vector of ones of size $(N+$ $1) \times 1$ and $\mathbf{I}$ is an identity matrix of size $(N+1) \times(N+1)$.

\section{Results and discussion}

We have presented a weakly nonlinear stability analysis of a rotating layer of a ferromagnetic fluid with temperature modulation at the boundary. We have obtained mathematical expressions for the stationary Rayleigh number $R a^{\alpha}$ and the magnetic Rayleigh number $N^{\alpha}$. Our results agree qualitatively with the results in $[1,9]$. To provide a measure of validation of our results we give a comparison with [9] in Tables 1 and 2 of the influence of the Taylor number on the critical wave number and the corresponding Rayleigh numbers. Although the results in the two studies are not directly comparable, of interest is the general trend observed, namely that in both cases, increasing $T a$ increases the critical wave number and the Rayleigh numbers suggesting that the influence of Taylor number is to stabilize the system.

The instability curves are given in Figures 1-3. Figure $1(a)-1(d)$ shows the influence of various parameters

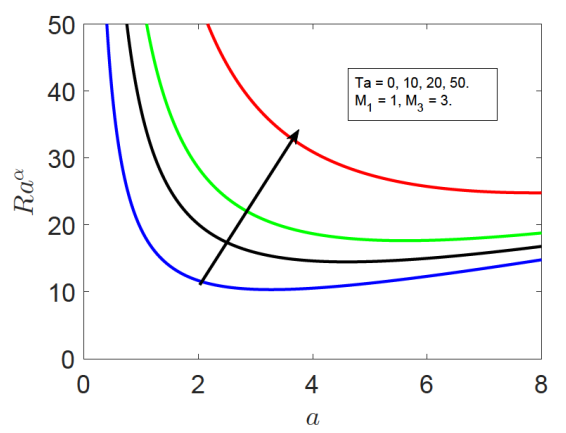

(a)

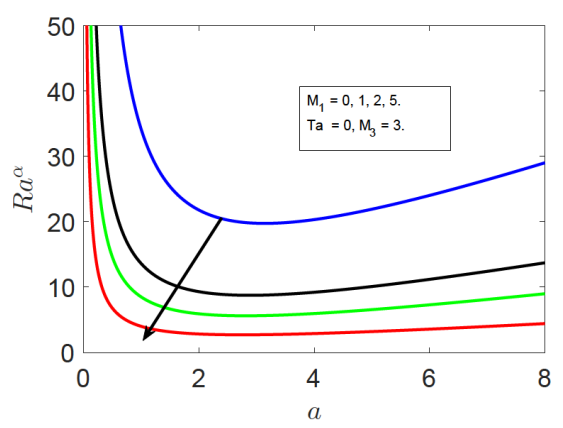

(b)

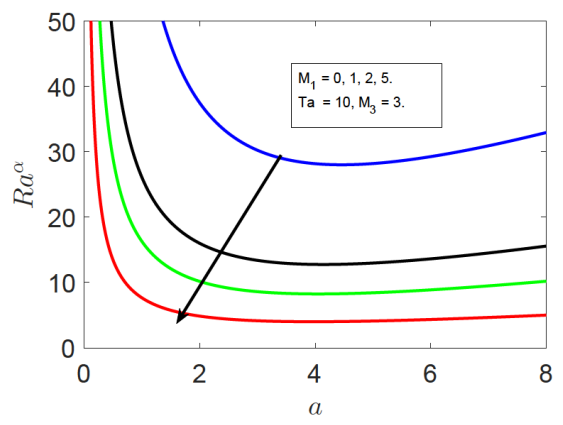

(c)

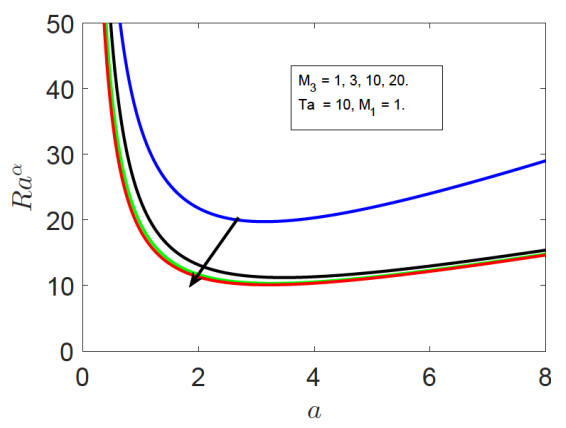

(d)

Figure 1: The effect of stationary Rayleigh number $R a^{\alpha}$ versus wave number $a$ for various values of (a) the Taylor number $T a$, (b)the ratio of magnetic force to the buoyancy force parameter $M_{1}$ with $T a=0$ (c) the ratio magnetic force to the buoyancy force parameter $M_{1}$ with $T a=10(\mathrm{~d})$ the nonlinearity of magnetization parameter $M_{3}$ 


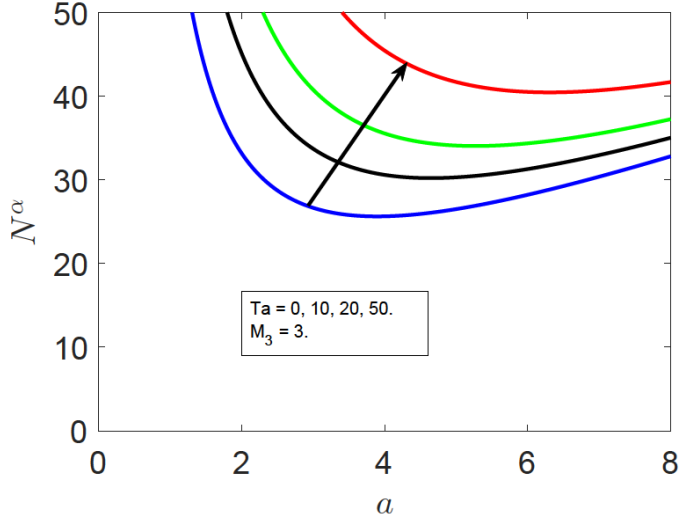

(a)

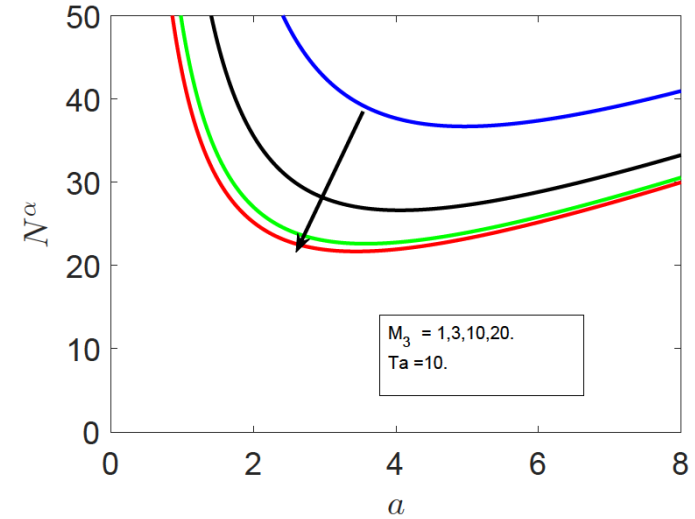

(b)

Figure 2: The effect of magnetic Rayleigh number $N^{\alpha}$ versus wave number $a$ for various values of (a) the Taylor number $T a$ (b) the nonlinearity of magnetization parameter $M_{3}$

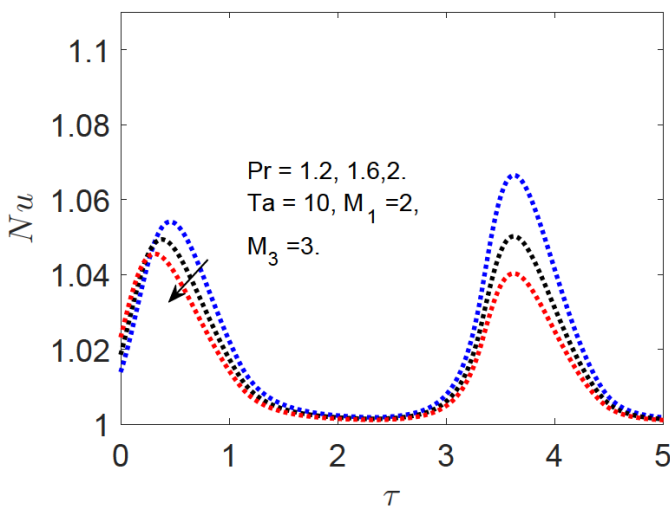

(a)

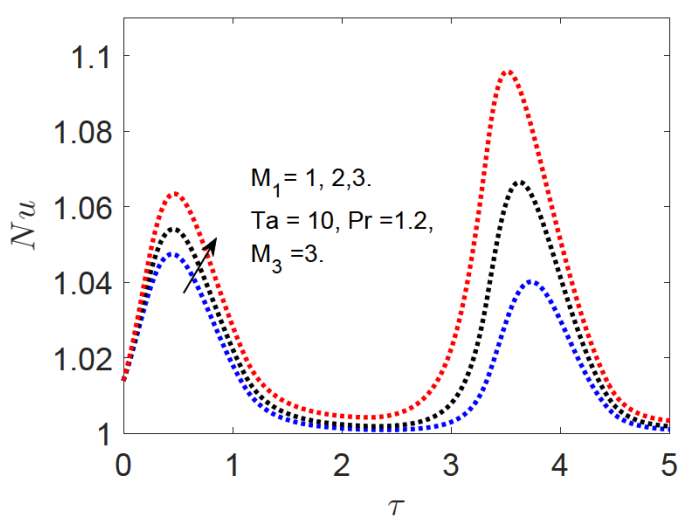

(c)

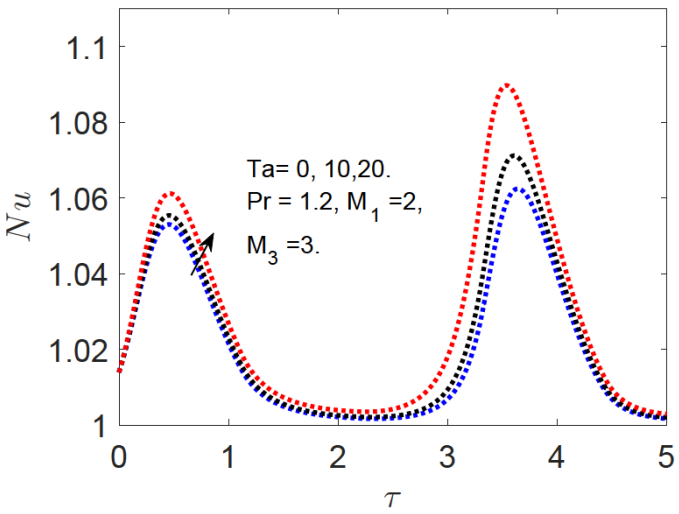

(b)

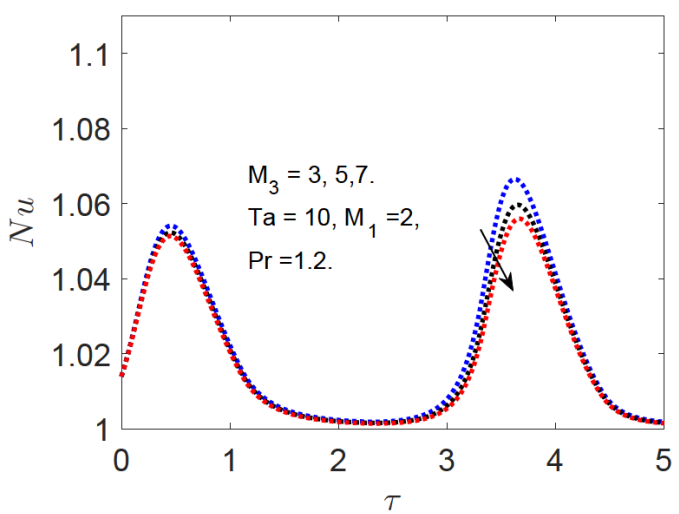

(d)

Figure 3: The variation of Nusselt number $N u$ with time $\tau$ in in-phase modulation ( $\phi=0)$ for different values of : (a) the Prandtl number $P r$, (b)the Taylor number $T a$, (c) the ratio of magnetic force to buoyancy force $M_{1}$ and (d) the nonlinearity of magnetization $M_{3}$

on the stationary Rayleigh number. It can be seen in Figures 1(a)and 2(a) that as Ta increases from 0 to 50 the values of the stationary Rayleigh and the magnetic Rayleigh numbers both increase. This shows that rotation has a stabilizing effect on the system. This result is similar to that of an ordinary viscous fluid. Rotation has a stabilizing influence on ferromagnetic fluid flow. Figures 1(b) and 1(c) show the relative influence of the size of the magnetic 


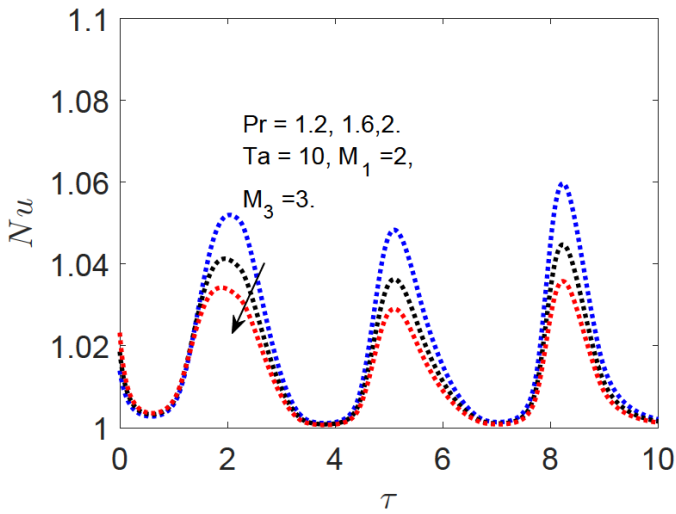

(a)

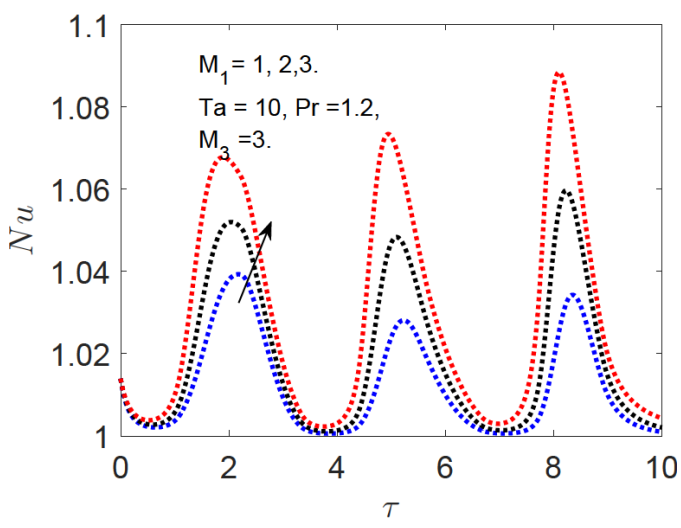

(c)

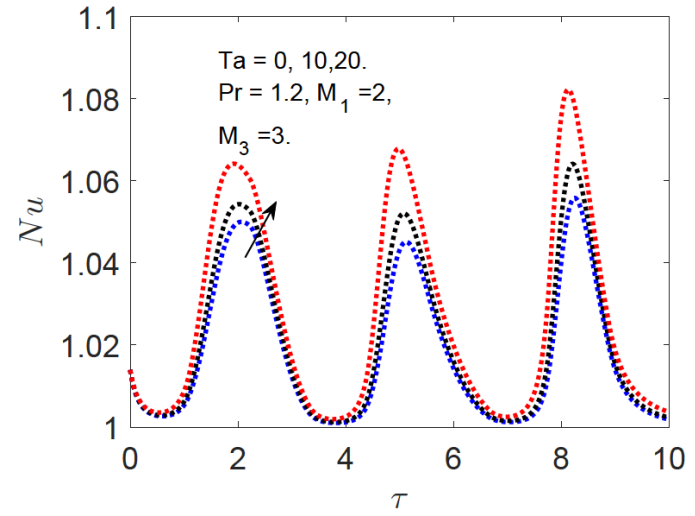

(b)

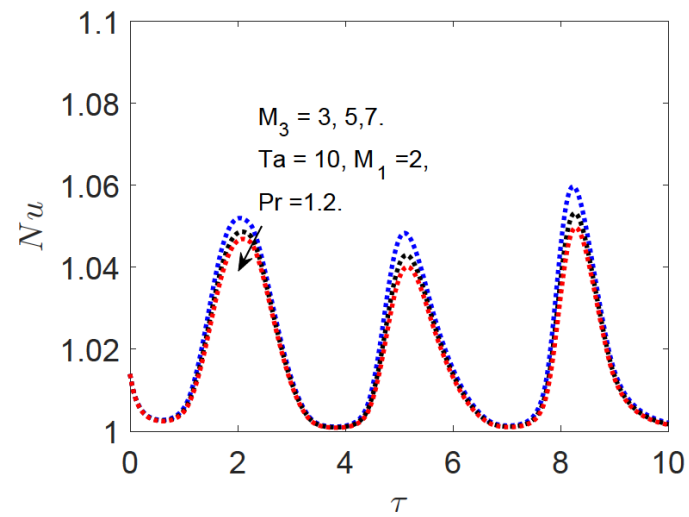

(d)

Figure 4: The variation of Nusselt number $N u$ with time $\tau$ in out-phase modulation $(\phi=\pi)$ for different values of : (a) the Prandtl number $P r$, (b)the Taylor number $T a$, (c) the ratio of magnetic force to buoyancy force $M_{1}$ and (d) the nonlinearity of magnetization $M_{3}$

force to the buoyancy force parameter $M_{1}$. As $M_{1}$ increases from 0 to 5 the stationary Rayleigh number is reduced. This suggest the magnetic and the buoyancy force are both destabilizing to the ferromagnetic fluid flow. Further, as $M_{1}$ increases with $T a=10$ fixed, the stationary Rayleigh number decreases, suggesting $M_{1}$ has a destabilizing effect for both low and high Taylor numbers. From Figures 1(d) and 2(b) it is observed that increasing $M_{3}$ from 1 to 20 reduces both the Rayleigh number and the magnetic Rayleigh number, this is destabilization to the system.

The Ginzburg-Landau equation is obtained using the nonlinear stability analysis at the third order of $\epsilon$. The equation was solved using a multi-domain spectral method. The heat transfer coefficient, represented by the Nusselt number, is presented graphically for in-phase and out-phase modulation in Figures 3-4. Figure 3(a)-3(d) show the effect of $\mathrm{Pr}, \mathrm{Ta}, M_{1}$ and $M_{3}$ on the Nusselt number with time $\tau$. It can be observed that on increasing the $\mathrm{Pr}$ and $M_{3}$, the Nusselt number decreases. Hence increasing these parameters reduces the rate of heat transfer. In- creasing $T a$ and $M_{1}$ increases the Nusselt number, thus the rate of heat transfer increases. Figures 4(a)-4(d) show changes in the Nusselt number with respect to time $\tau$ due to the influence of various parameters in the case of out of phase modulation. It can be observed that the Nusselt number for in-phase modulation is less than for out of phase modulation.

A multi-domain spectral collocation method was used to find the nonlinear amplitudes in ferromagnetic fluid convection equations for various values of $R$. The solution sets were obtained using initial conditions selected in the neighborhood of the stationary points corresponding to the motionless solutions. The simulations were done to a maximum time $\tau_{\max }=20$. For a sense of the accuracy of the method, the solutions were compared with solutions obtained using the Runge-Kutta based ode 45 routine. Figures 5(a)-5(d) show the time series solution of $X_{1}(\tau)$ for different supercritical values of $R$. As $R$ increases, periodic solutions are obtained. Here a comparison between the multi-domain spectral collo- 

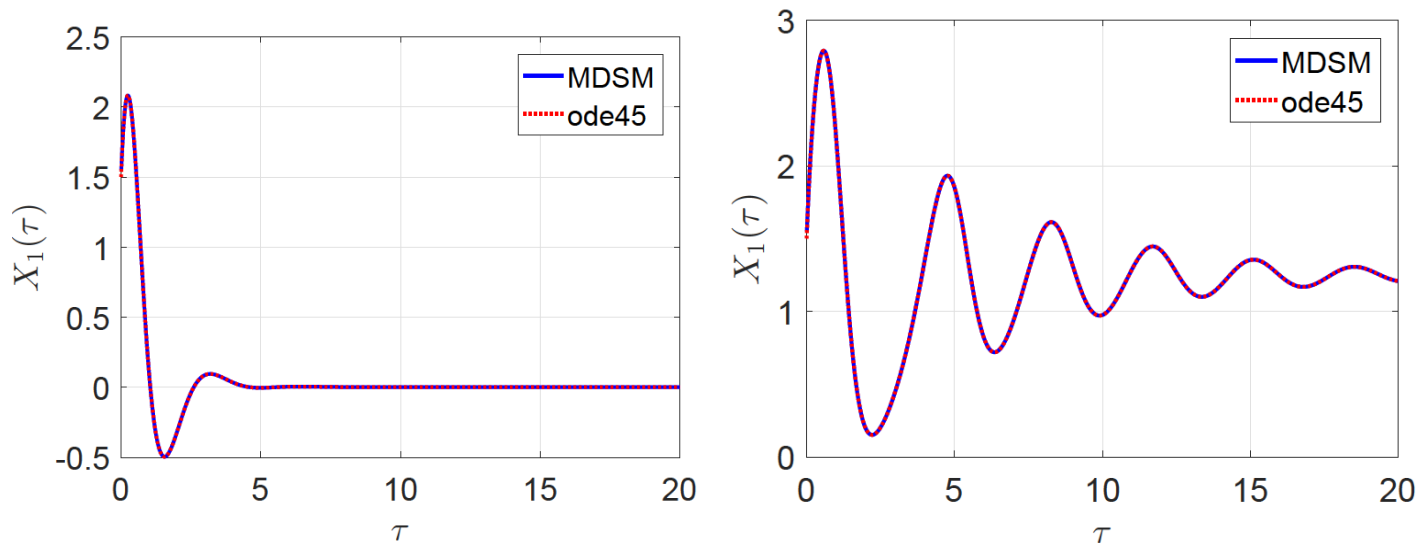

(a)

(b)
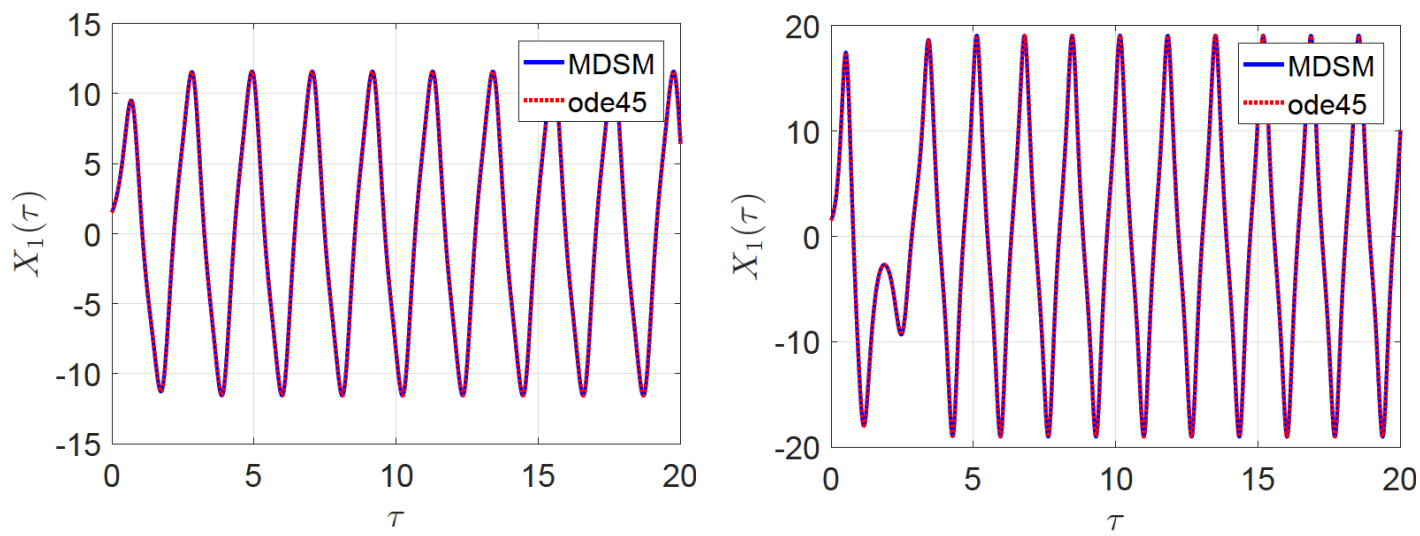

(c)

(d)

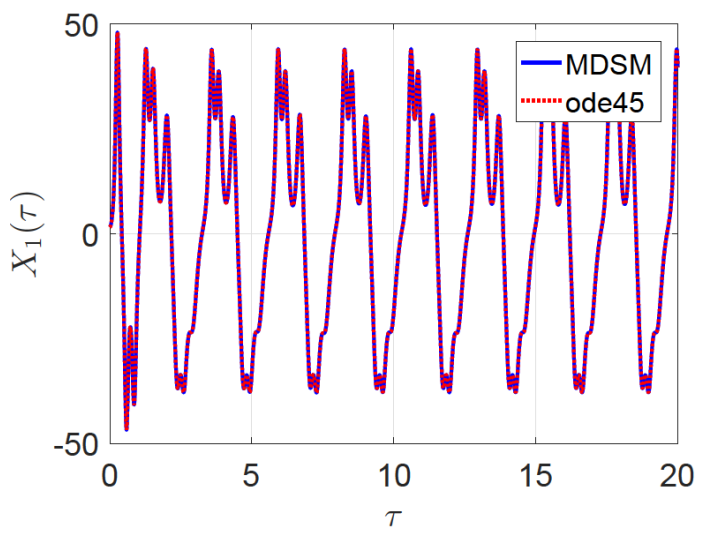

(e)

Figure 5: Comparison between a Multi-domain spectral collocation methods and ode45 methods for the solution of $X_{1}(\tau)$ for different values of $R$ (a) $R=2$, (b) $R=4$, (c) $R=10$, (d) $R=20$ and (e) $R=100$

cation method and the ode45 is given. In Figures 611 we present a projection of the trajectories onto the $\left(X_{1}, X_{2}\right),\left(X_{1}, X_{3}\right),\left(X_{1}, X_{4}\right),\left(X_{2}, X_{3}\right),\left(X_{2}, X_{4}\right)$ and $\left(X_{3}, X_{4}\right)$ phase planes, respectively. The initial supercritical convective solution $R=2$ is presented in part $a$ in each figure. We observe that the trajectories attracted to equilib- rium points that correspond to the motionless solution are stable spirals. The solutions are presented in part $c$ and $d$ of each figure when $R=20$ and $R=25$, respectively. For these Rayleigh numbers, chaotic solutions are obtained. These changes in solutions are further presented in Figures 6(c)-6(d), 7(c)-7(d) and 9(c)-9(d). The results pre- 


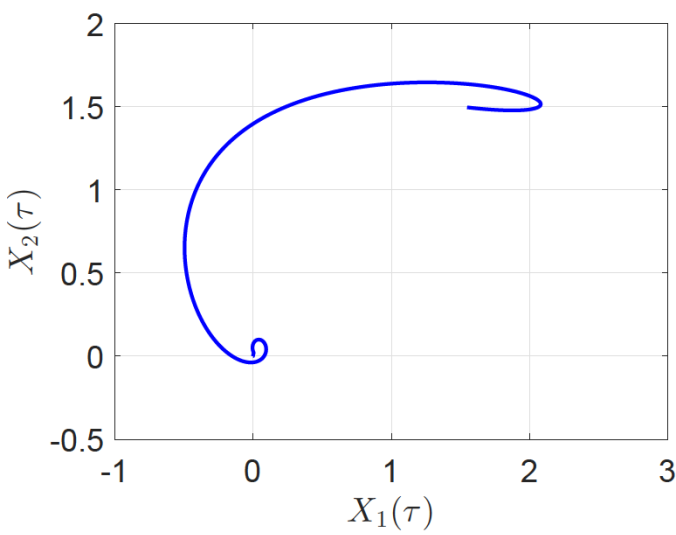

(a)

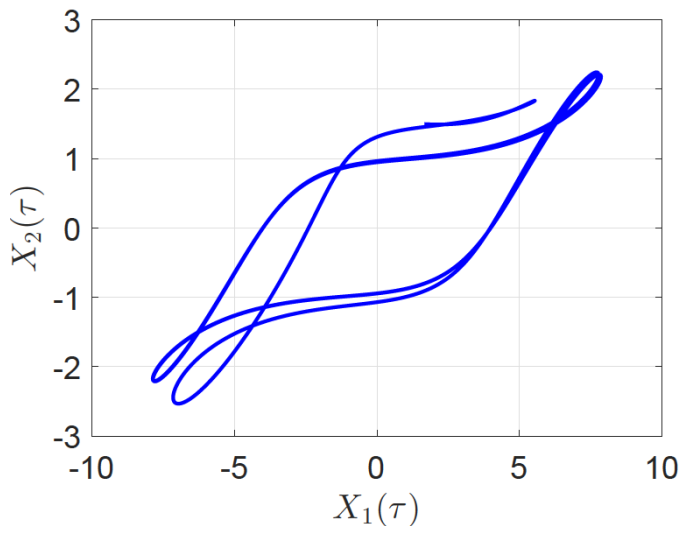

(c)

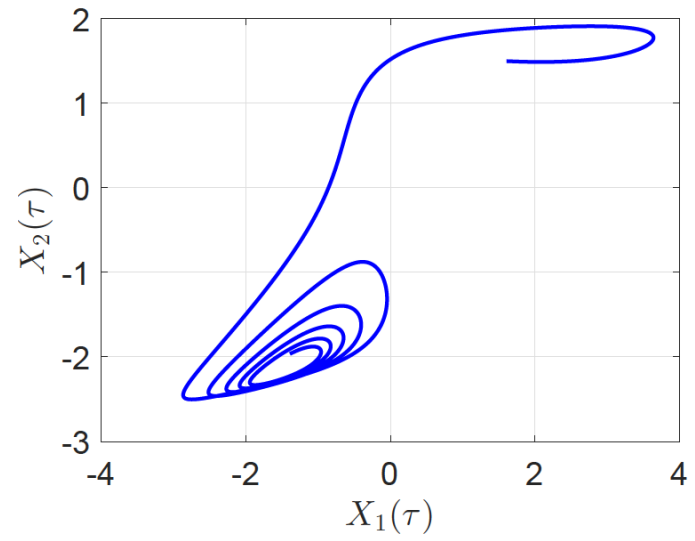

(b)

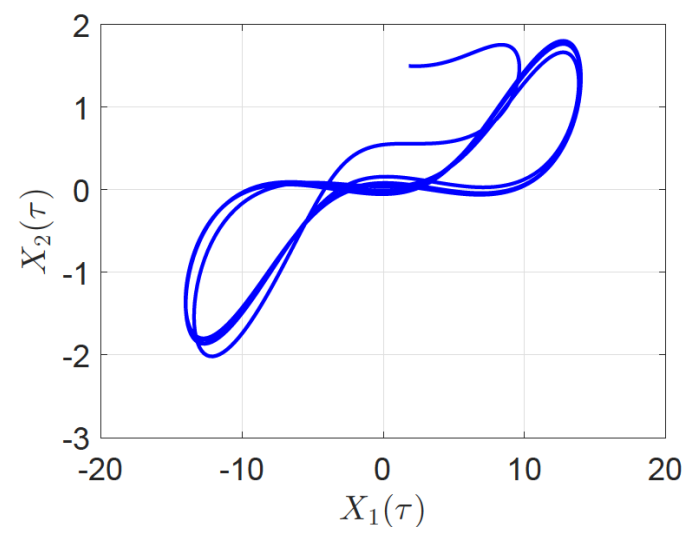

(d)

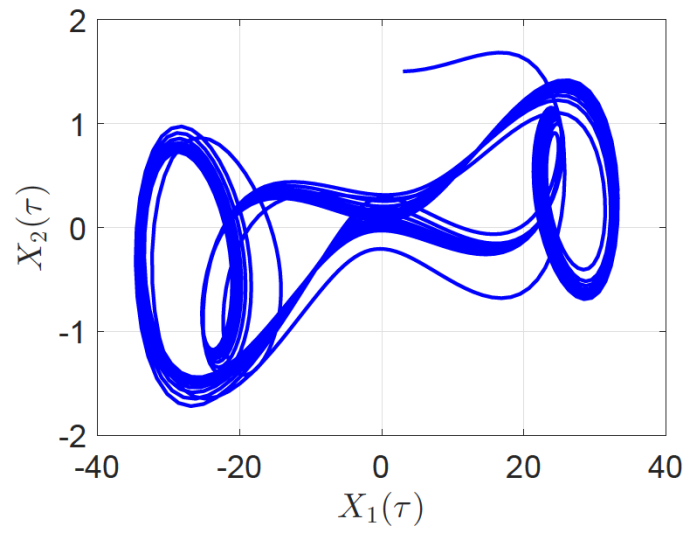

(e)

Figure 6: The evolution of trajectory over times in phase space for increasing values of Rayleigh number.( in term of $R$ ) corresponding to (a) $R=2$, (b) $R=4$, (c) $R=10$, (d) $R=20$ and (e) $R=100$. The graph represented the projection of the solution into $X_{1} X_{2}$ plane

sented in Figures 8(c)-8(d) and 10(c)-10(d) show a transition to a limit cycle. Increasing the values of $R$, for example, when $R=100$ the results are complex with a significant level of unpredictability.

Figure 12 shows the streamlines patterns for the flow of a ferromagnetic fluid. Two different eddies are observed.
The clockwise and anti-clockwise flows are shown via negative and positive stream function values, respectively. With the Rayleigh number increasing from 2 to 200, the magnitude of the stream function values increase. The sense of motion in the subsequent cells is opposite that of an adjoining cell, indicating symmetry in the forma- 


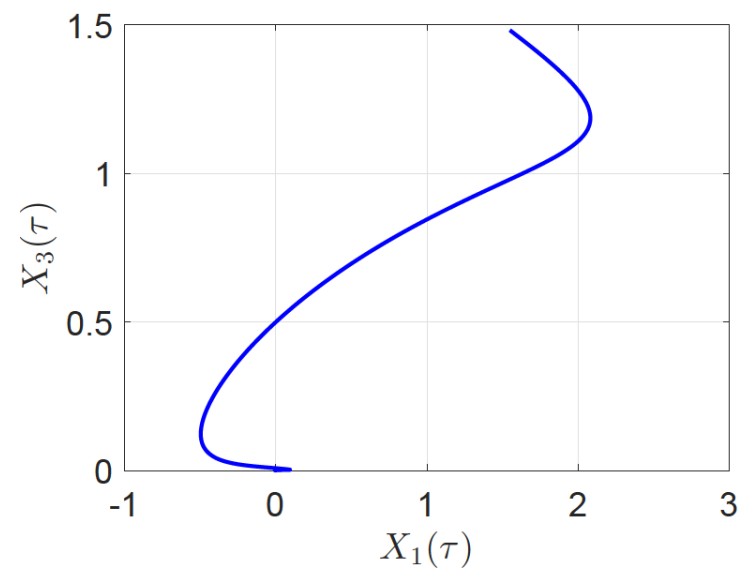

(a)

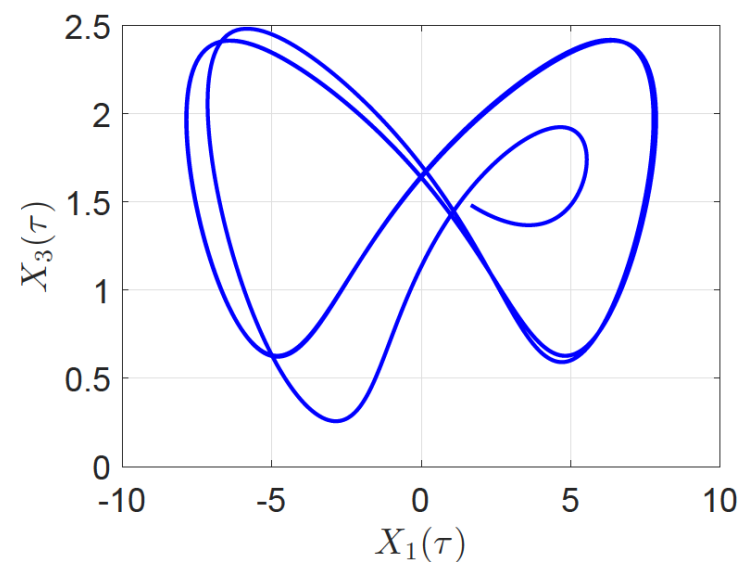

(c)

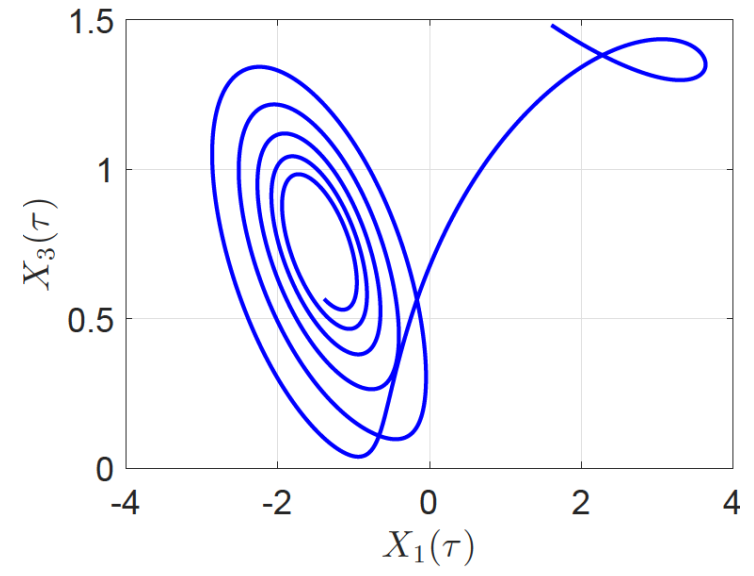

(b)

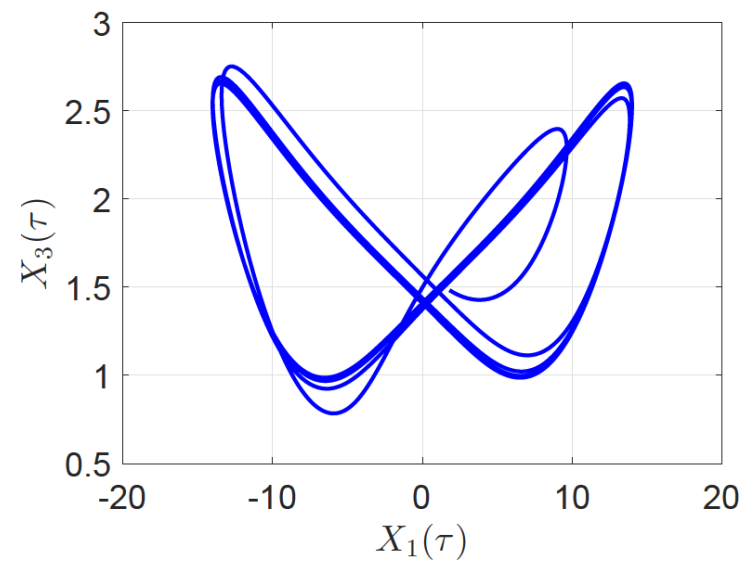

(d)

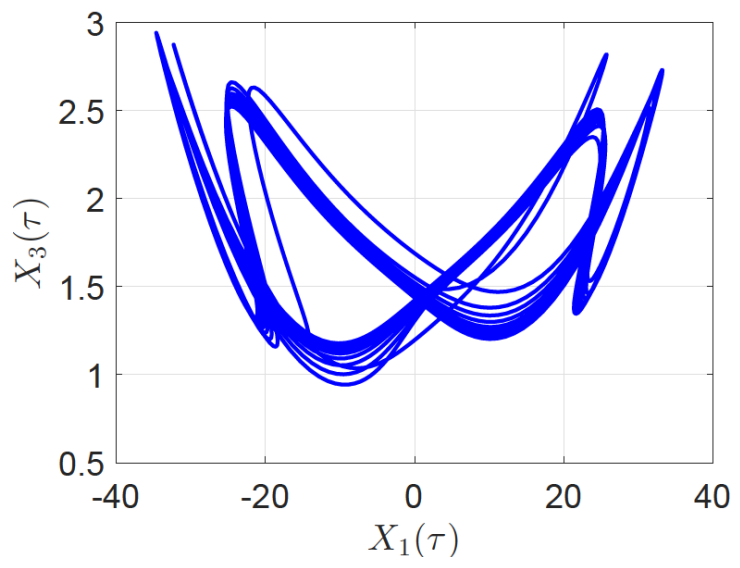

(e)

Figure 7: The evolution of trajectory over times in phase space for increasing values of Rayleigh number. (in term of $R$ ) corresponding to (a) $R=2$, (b) $R=4$, (c) $R=10$, (d) $R=20$ and (e) $R=100$. The graph represented the projection of the solution into $X_{1} X_{3}$ plane

tion of ferromagnetic convective cells. Figure 13 shows the isotherm patterns as the Rayleigh number changes from 2 to 200. Three different eddies are observed. The small eddy at the left corner diminishes as $R$ increases from 2 to 200 .
Also, increasing $R$ reduces the density of the isotherms implying a delay of the onset of instability. 


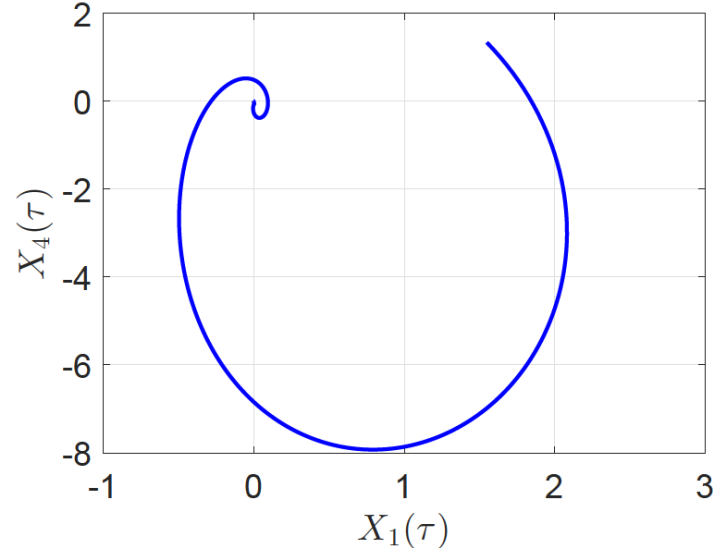

(a)

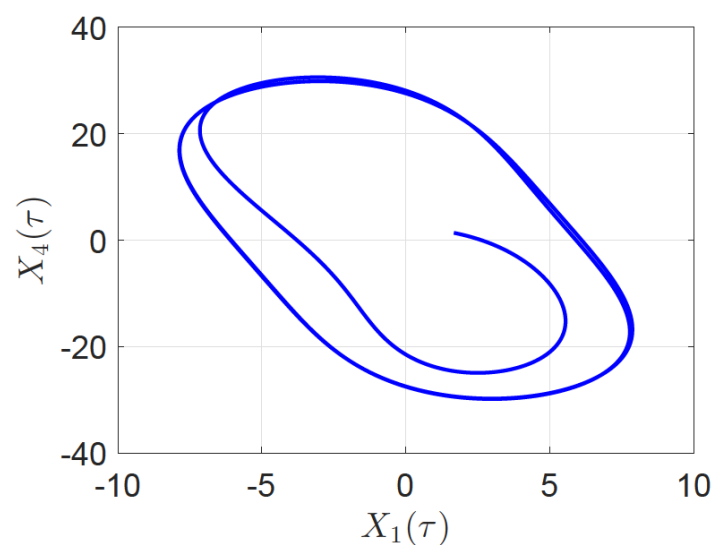

(c)

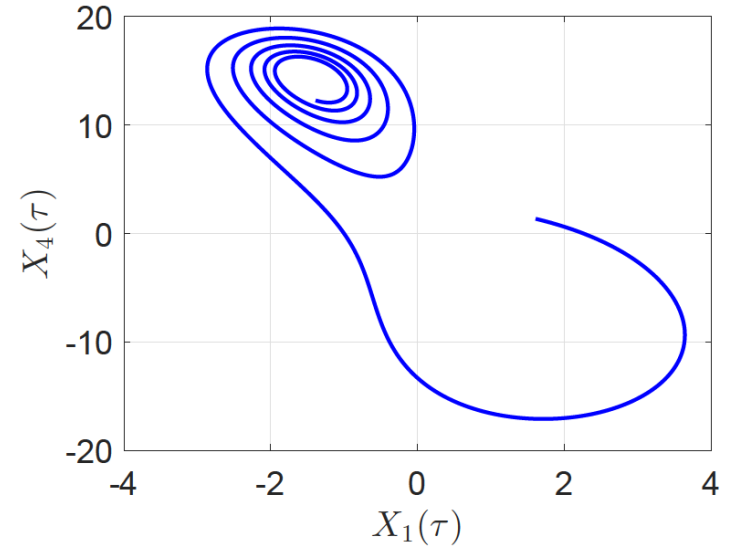

(b)

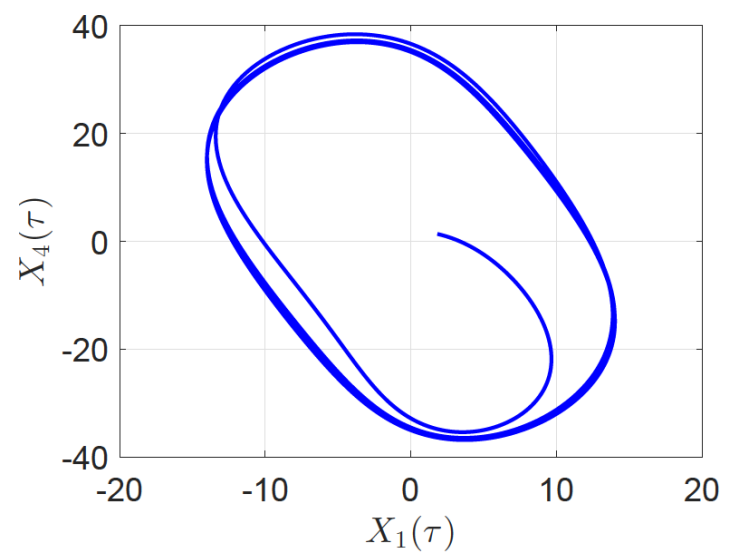

(d)

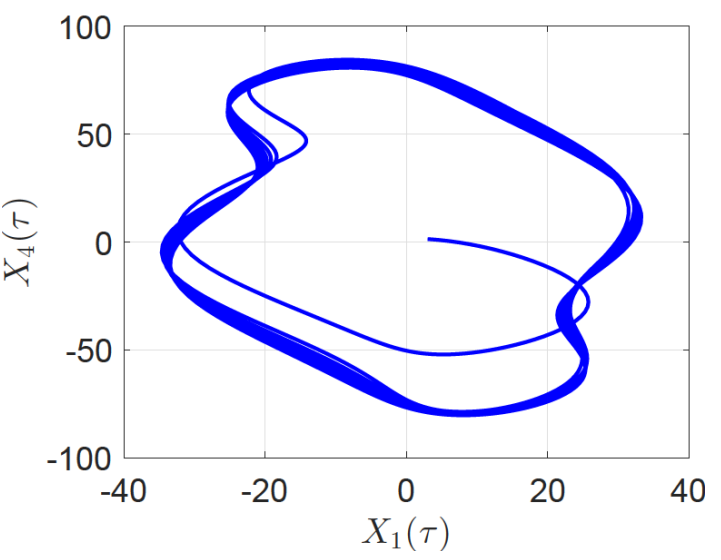

(e)

Figure 8: The evolution of trajectory over times in phase space for increasing values of Rayleigh number. (in term of $R$ ) corresponding to (a) $R=2$, (b) $R=4$, (c) $R=10$, (d) $R=20$ and (e) $R=100$. The graph represented the projection of the solution into $X_{1} X_{4}$ plane 


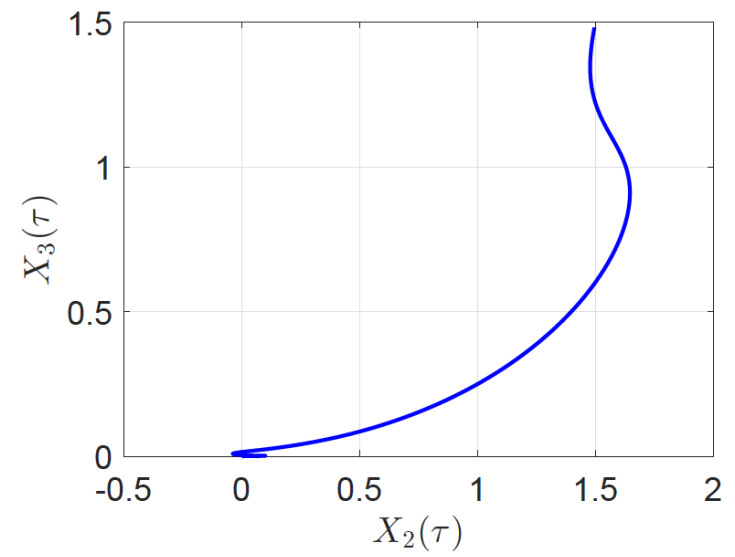

(a)

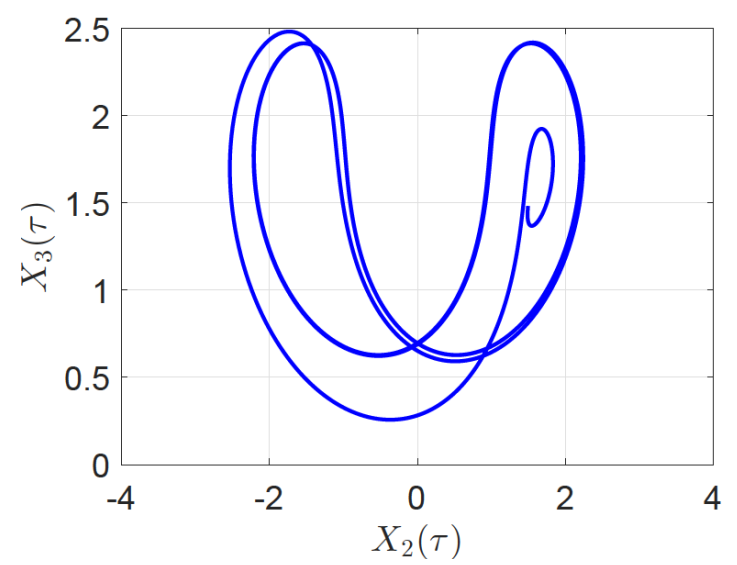

(c)

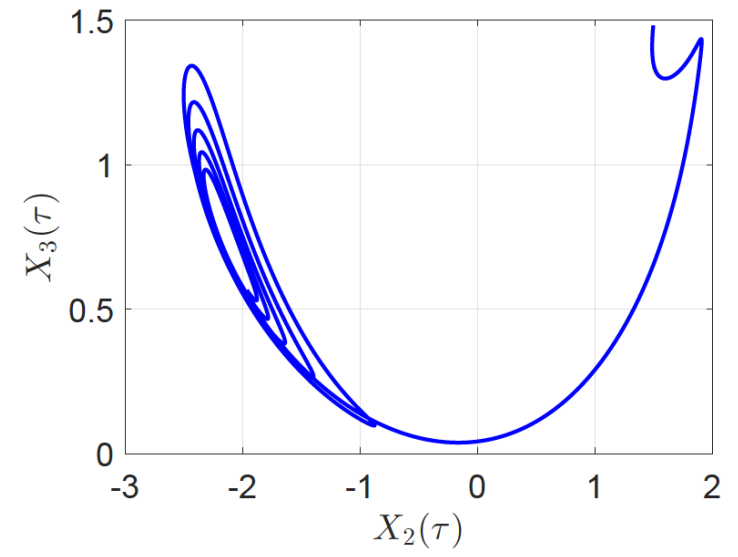

(b)

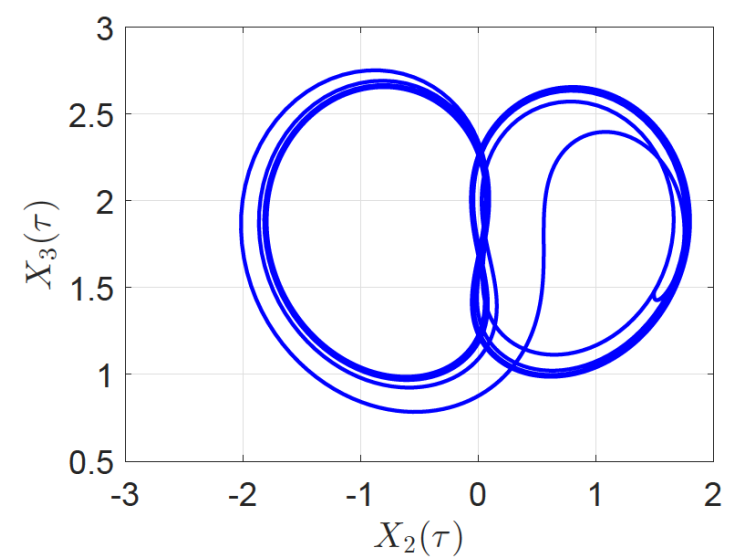

(d)

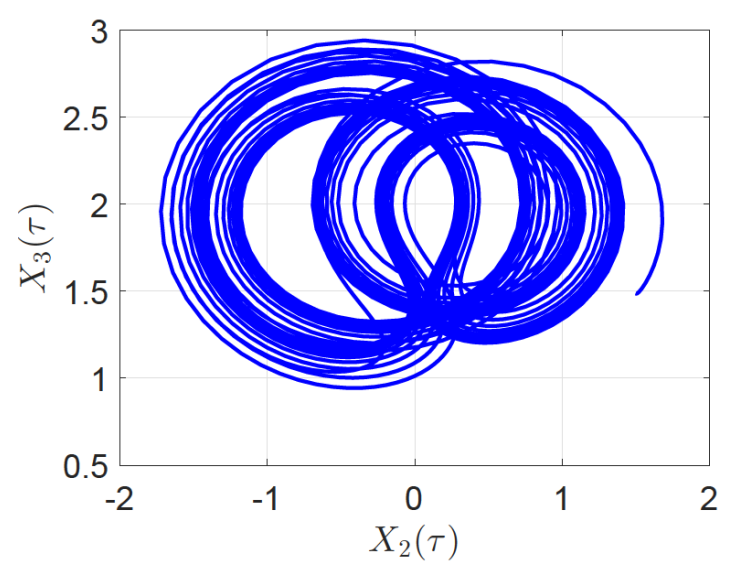

(e)

Figure 9: The evolution of trajectory over times in phase space for increasing values of Rayleigh number. (in term of $R$ ) corresponding to (a) $R=2$, (b) $R=4$, (c) $R=10$, (d) $R=20$ and (e) $R=100$. The graph represented the projection of the solution into $X_{2} X_{3}$ plane 


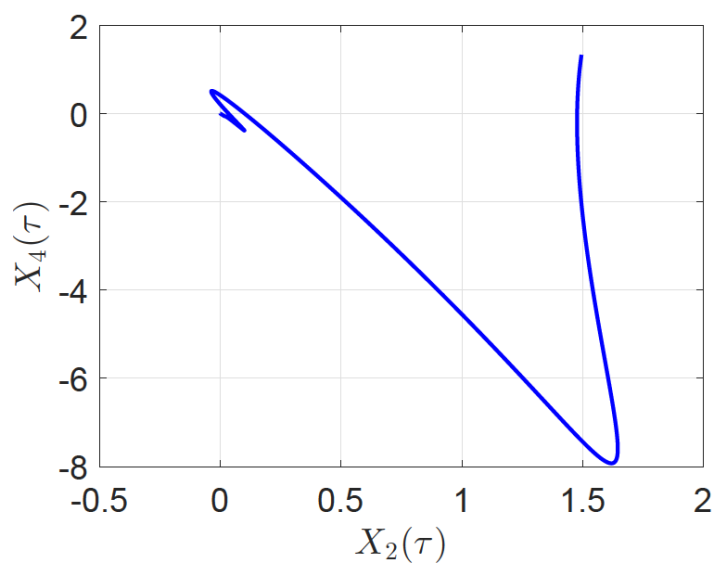

(a)

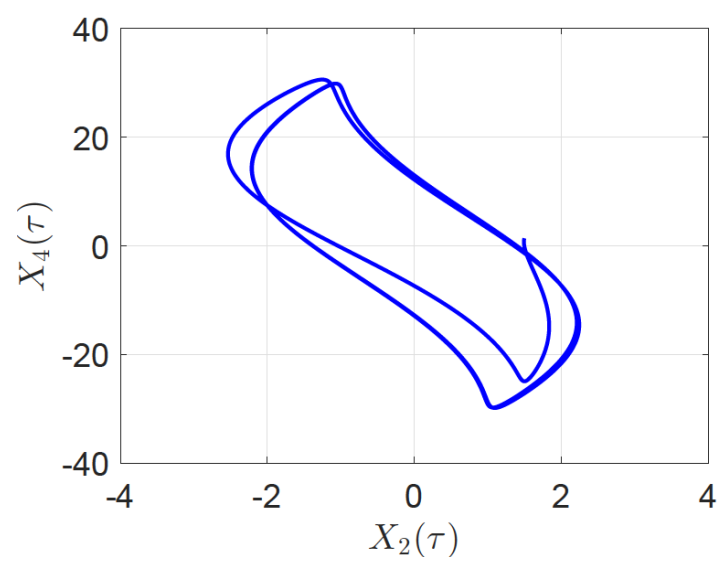

(c)

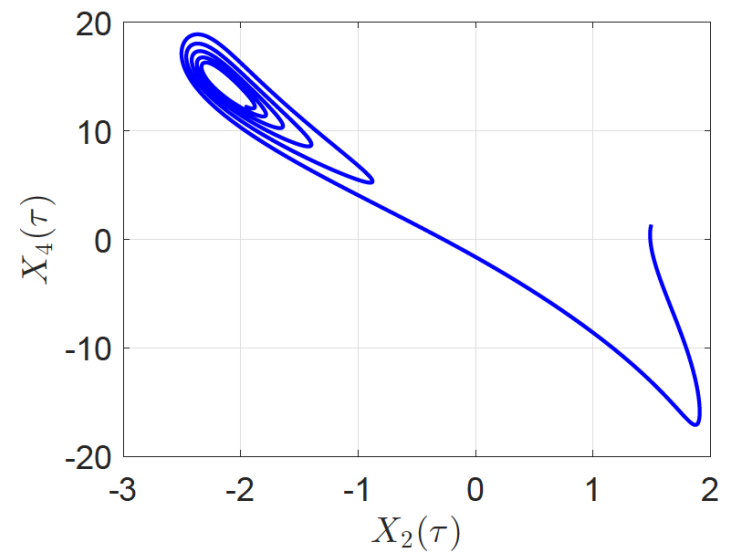

(b)

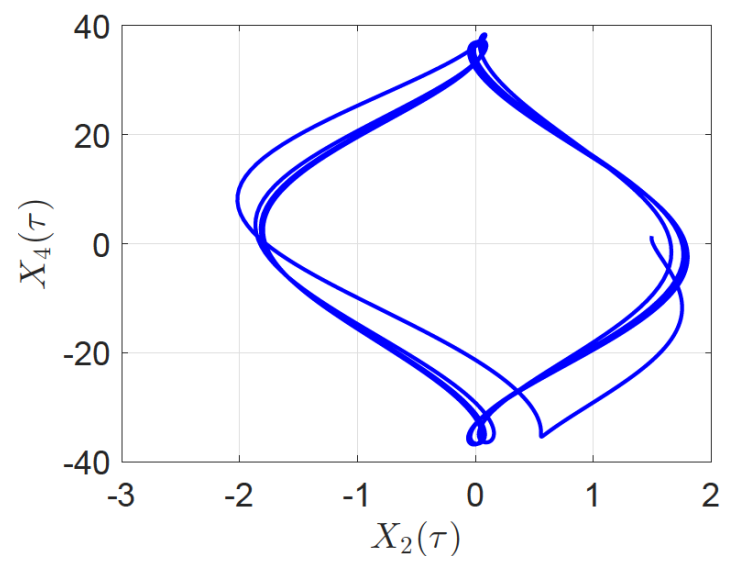

(d)

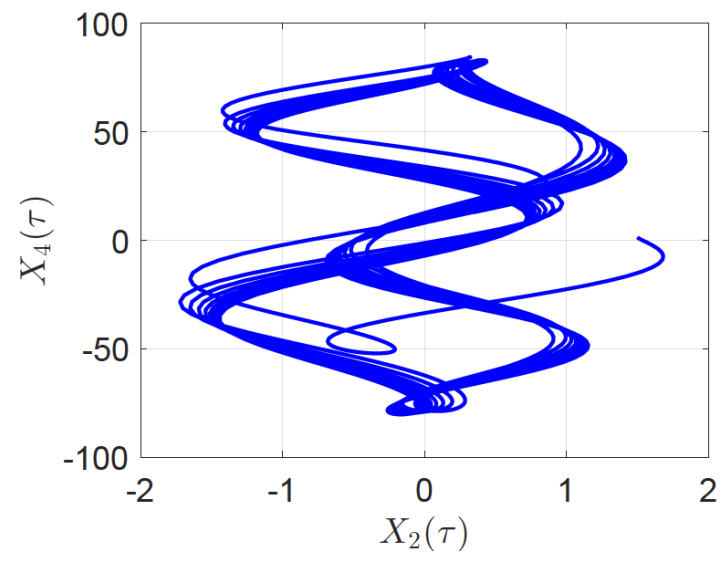

(e)

Figure 10: The evolution of trajectory over times in phase space for increasing values of Rayleigh number. (in term of $R$ ) corresponding to (a) $R=2$, (b) $R=4$, (c) $R=10$, (d) $R=20$ and (e) $R=100$. The graph represented the projection of the solution into $X_{2} X_{4}$ plane 


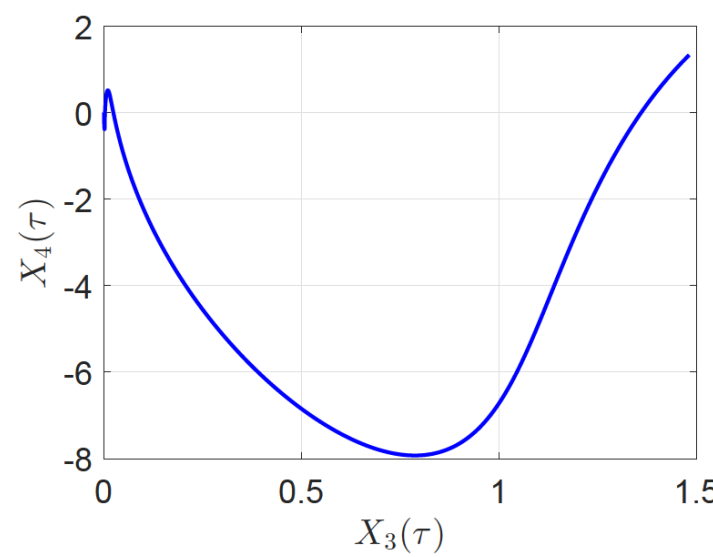

(a)

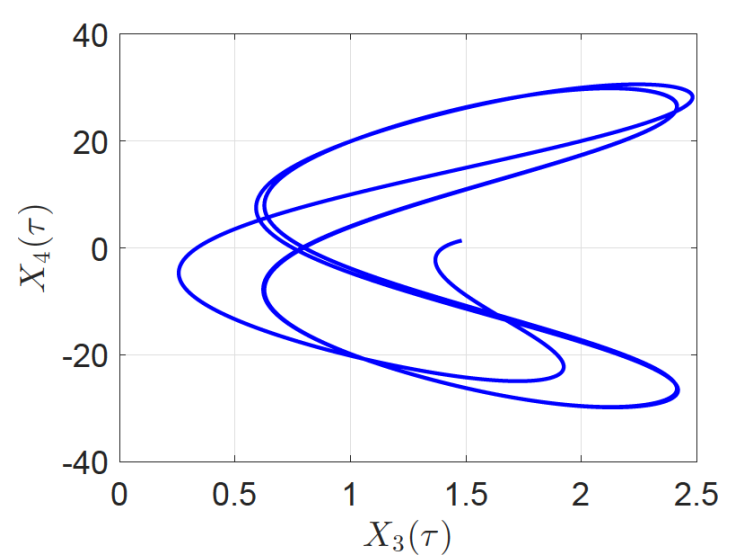

(c)

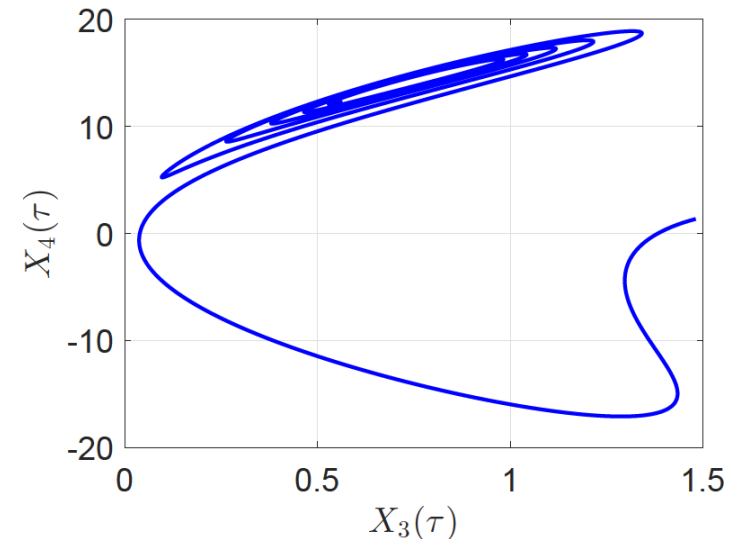

(b)

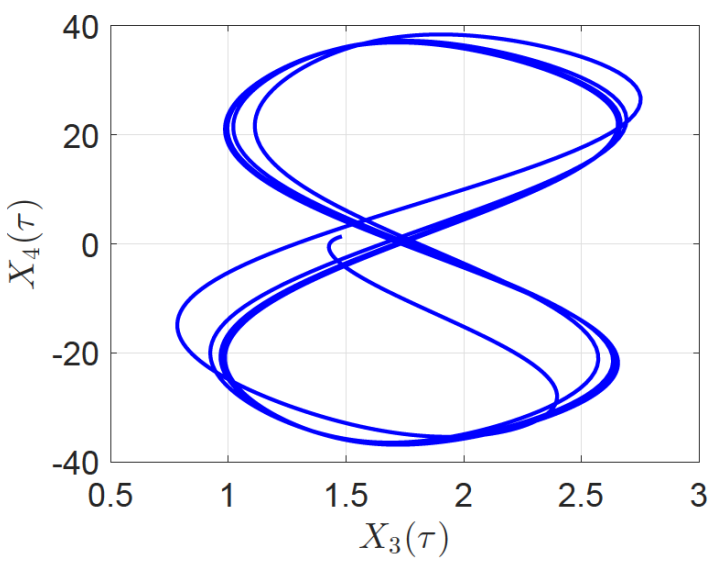

(d)

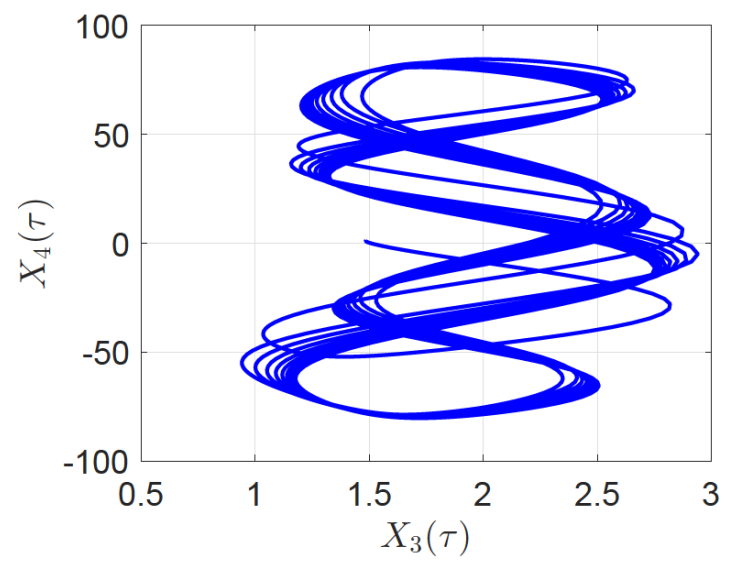

(e)

Figure 11: The evolution of trajectory over times in phase space for increasing values of Rayleigh number. (in term of $R$ ) corresponding to (a) $R=2$, (b) $R=4$, (c) $R=10$, (d) $R=20$ and (e) $R=100$. The graph represented the projection of the solution into $X_{3} X_{4}$ plane 


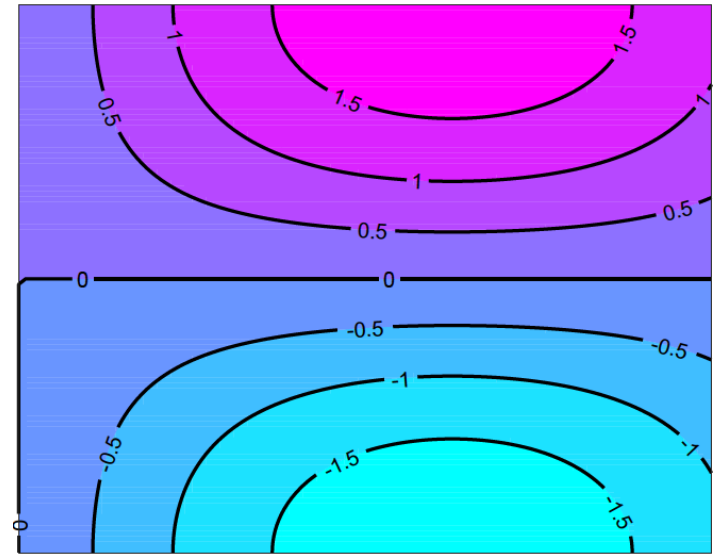

(a)

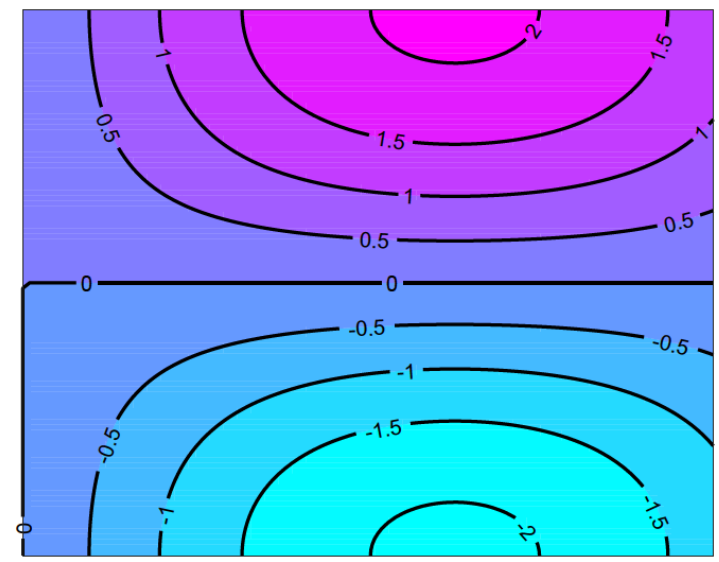

(c)

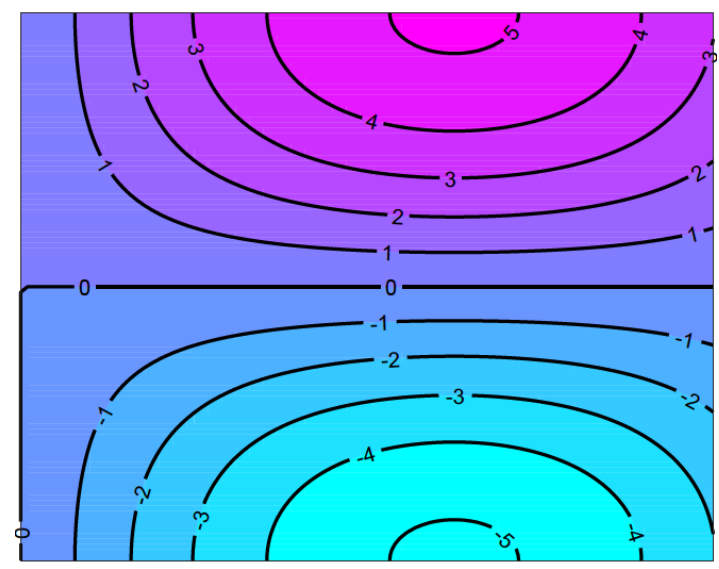

(e)

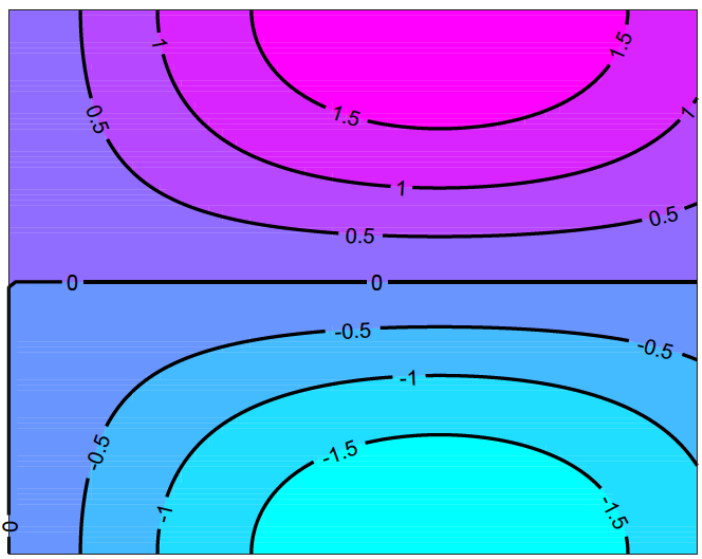

(b)

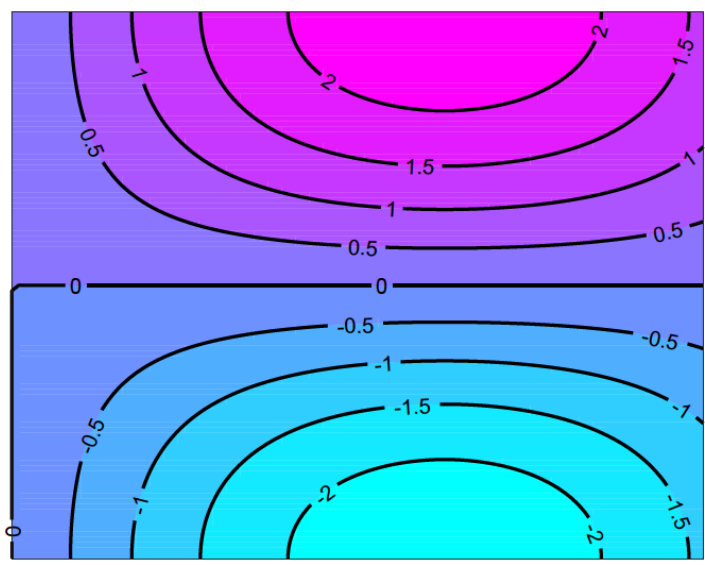

(d)

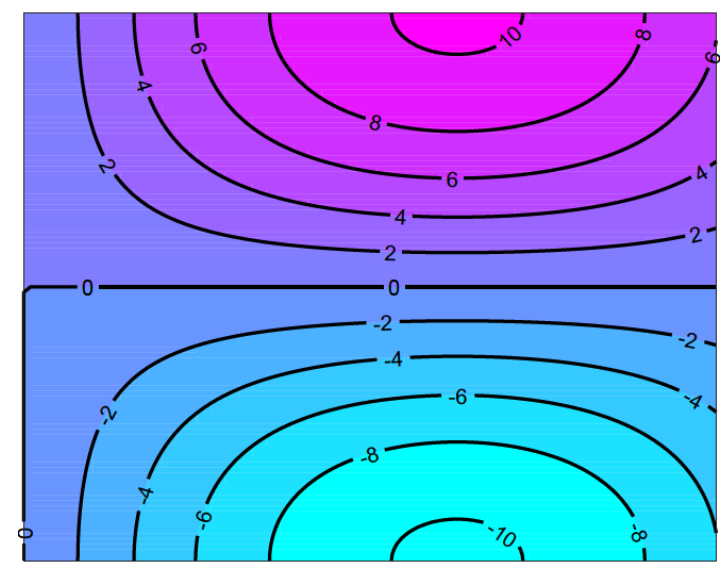

(f)

Figure 12: The streamlines for different values of the Rayleigh number $R$ e.g. $R=2,4,10,20,100,200$ 


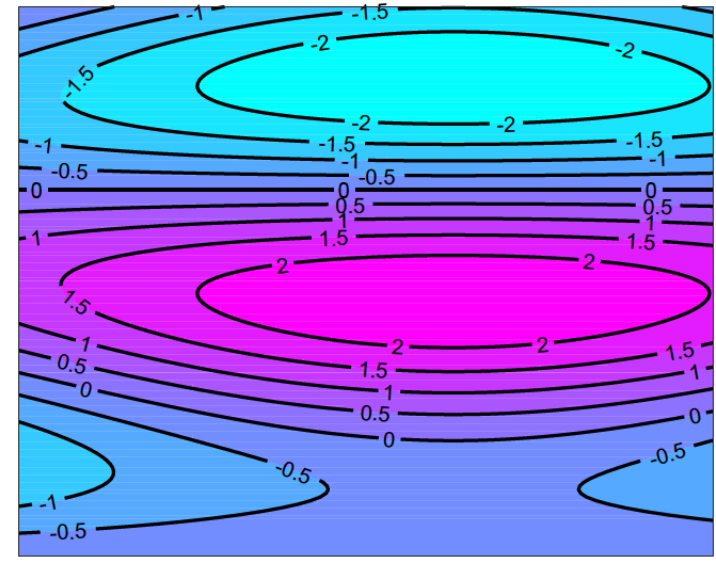

(a)

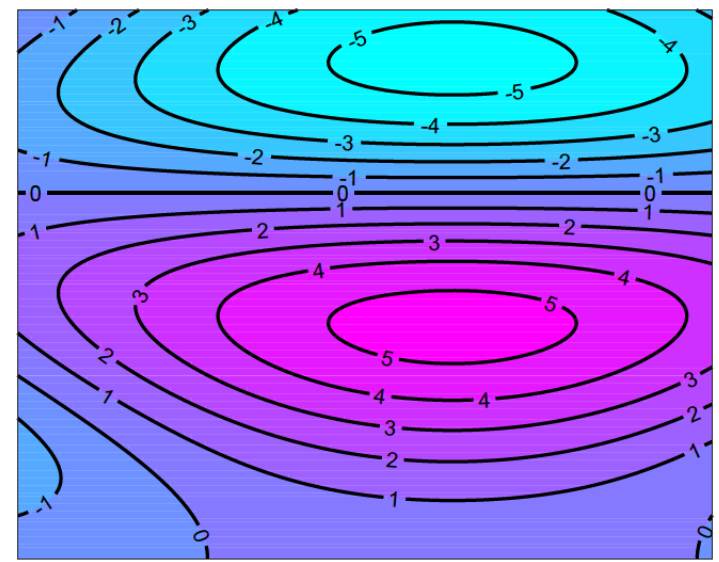

(c)

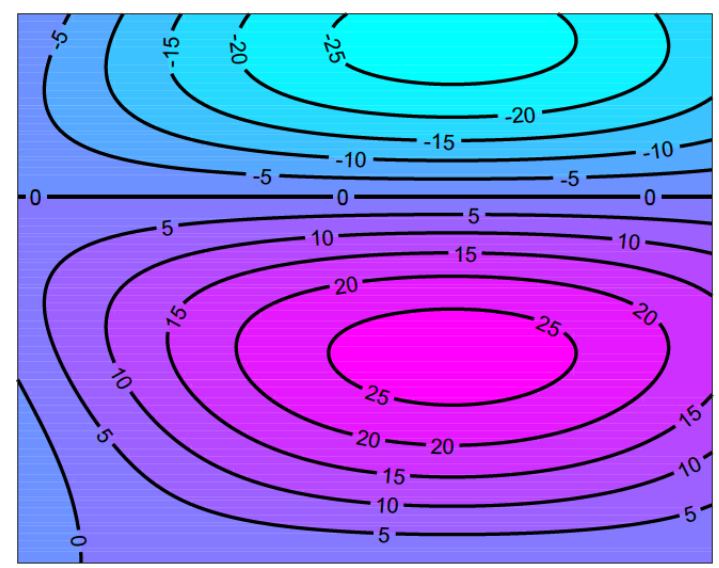

(e)

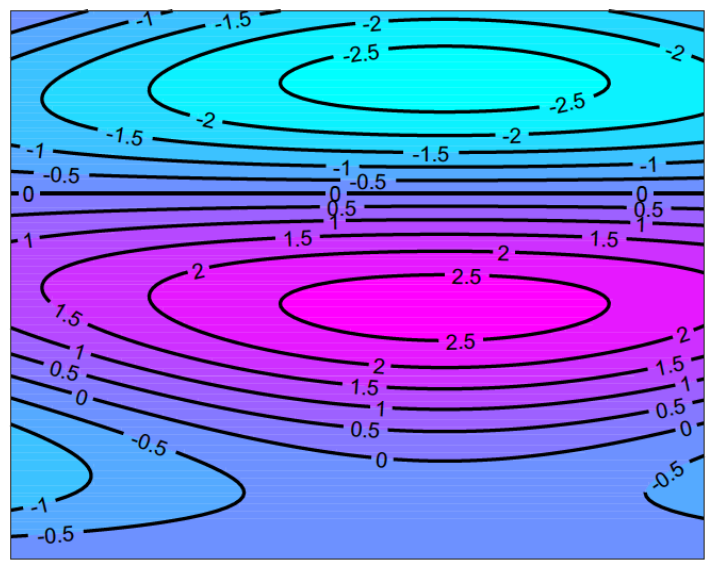

(b)

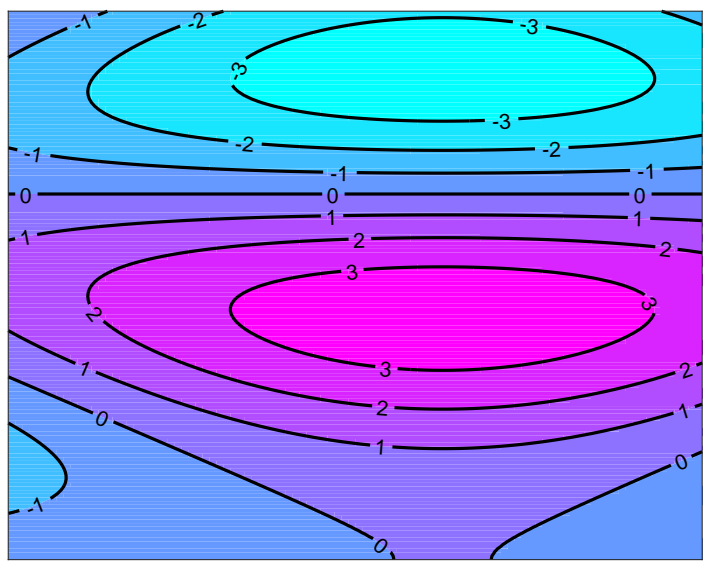

(d)

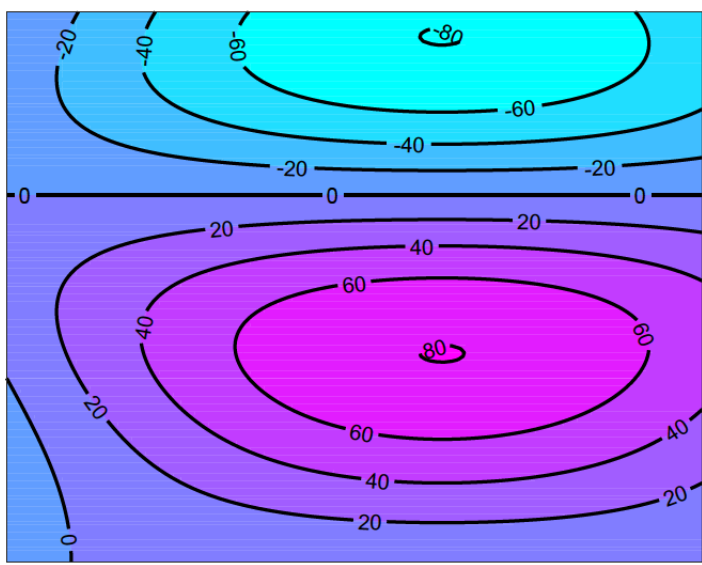

(f)

Figure 13: The isotherms for different values of the Rayleigh number $R$ e.g. $R=2,4,10,20,100,200$ 


\section{Conclusion}

We investigated the thermoconvective instability in a rotating ferromagnetic fluid layer with time periodic temperature boundary conditions. The influence of flow parameters such as the Rayleigh number on the onset of instability was determined using a weakly nonlinear stability analysis. The results are broadly in line with the earlier findings in $[1,3,8]$. The heat transport has been analyzed for both the in-phase and out of phase temperature modulations. The influence of the parameters such as the Prandtl number, Taylor number and the magnetization parameter on the Nusselt number for the in-phase modulation was found to be less significant compared to the case of out of phase modulation.

The set of nonlinear differential equations for convection amplitude was solved using a multi-domain spectral collocation method. The accuracy of the solutions was determined by comparison with solutions using a different independent method, namely the Runge-Kutta based ode45 Matlab solver. The stability of the equilibrium solutions of the nonlinear differential equations has been analyzed. Transitions from different states have been demonstrated for different parameter values, for example from steady convection to chaotic solutions at high Rayleigh numbers.

\section{References}

[1] Finlayson, B.A., Convective instability of ferromgnetic fluids, J. Fluid Mech., 1970, 40(4), 755-767.

[2] Chandrasekhar, S., Hydrodynamic and hydromagnetic stability, 1961, Oxford University Press, Oxford.

[3] Rosensweig, R.E.,Ferrohydrodynamics, 1985, Cambridge University Press.

[4] Schwab L., Hildebrandt U., Stierstadt K., Magnetic Benard convection, J. Magn. Magn. Mat., 1983, 39(1-2), 113-114.

[5] Stiles P.J., Kagan M., Thermoconvective instability of a horizontal layer of ferrofluid in a strong magnetic field, J. Magn. Magn. Mat., 1990, 85(1-3), 196-198.

[6] Ruessll C.L., Blnnerhassett P.J., Stiles P.J., Large wave number convection in magnetized ferrofluids, J. Magn. Magn. Mat., 1995, 149(1-2), 196-121.
[7] Greenspan H.P., The theory of rotating fluids, 1968, Cambridge University press, London.

[8] Gupta M.D., Gupta A.S., Convective instability of a layer of a ferromagnetic fluid rotating about a vertical axis, Int. J. Eng, Sci., 1979, 17(3), 271-277.

[9] Venkatasubramanian S., Kaloni P.N., Effects of rotation on the thermo-convective instability of a layer of a ferrofluids, Int. J. Eng. Sci., 1994, 32(2), 237-256.

[10] Gotoh K., Yamada M., Thermal convective in a horizontal layer of magnetic fluid, J. Phys. Soc. Japan, 1982, 51(9), 3042-3048.

[11] Bhasauria B.S., Siddheshwar P.G., Kumar J., Suthar O.P., Weakly nonlinear stability analysis of temerature/gravity modulated stationary Rayleigh-Benard convection in a rotating porous medium, Transp. Porous Med., 2012,92(3), 633-647.

[12] Rudraiah N., Sekhar N.G., Convective on magnetic fluids with internal heat generation, ASME J. Heat Transfer, 1991, 113(1), 122127.

[13] Aniss S., Belhaq M., Souhar M., Effects of amagnetic mod- ulation on the stability of a magnetic liquid layer heated from above, J. Heat Transfer, 2001,123(3) 428-432.

[14] Singh J., Bajaj R., convective instability ina ferrofluids layer with temperature modulation rigid boundaries, Fluid Dyn. Res., 2011, 43(2), 025502.

[15] Trefethen L.N., Spectral methods in MATLAB, SIAM, Philadelphia, 2000, 26(2), 199

[16] Motsa S.S., Dlamini P., Khumalo M., A new multistage spectral relaxation method for solving chaotic initial value systems, Nonlin. Dyn., 2013, 72(1-2) 265-83.

[17] Motsa S.S., Magagula V.M., Goqo P.S., Oyelakin I.S., Sibanda P., A multi-domain spectral collocation approach for solving LaneEmden type equations, Chaper 7, Numerical Simulation - From Brain Imaging to Turbulent flows, 2016, 10, 5772-63016.

[18] Noreldin O.A.I., Sibanda P., Mondal S., Weakly Nonlinear Stability in a Horizontal Porous Layer Using a Multi-domain Spectral Collocation Method, chapter 9, Complexity in Biological and Physical Systems, 2018, 71066.

[19] Bhadauria B.S., Fluid convection in a rotating porous layer under modulated temperature on the boundaries, Transp. Porous Med., 2007, 67 297-315.

[20] Mielke A., The Ginzburg-Landau Equation in Its Role as a Modulation Equation, Chapter 15, Handbook of Dynamical Systems, 2002, 2, 759-834.

[21] Routh-Hurwitz criterion, Encyclopedia of Mathematics, http: //www.encyclopediaofmath.org/index.php?title=Routh-Hurwit z_criterion\&oldid $=33371$ 